\title{
Mutational topography reflects clinical neuroblastoma heterogeneity
}

\section{Anton Henssen ( $\square$ anton.henssen@charite.de )}

Charité https://orcid.org/0000-0003-1534-778X

\section{Elias Rodriguez-Fos}

Charité - Universitätsmedizin Berlin

\section{Martin Burkert}

Centre for Molecular Medicine Norway, University of Oslo https://orcid.org/0000-0002-6721-9985

\section{Montserrat Puiggròs Maldonado}

Barcelona Supercomputing Center https://orcid.org/0000-0001-5034-7924

\section{Joern Toedling}

Charité - Universitätsmedizin Berlin https://orcid.org/0000-0003-1471-1295

\section{Nina Thiessen}

$\mathrm{BIH}$

\section{Eric Blanc}

Charité - Universitätsmedizin Berlin, Corporate Member of Freie Universität Berlin, Humboldt-Universität zu Berlin, and Berlin Institute of Health, Core Unit Bioin

\section{Annabell Szymansky}

Charité-Universitätsmedizin Berlin

\section{Falk Hertwig}

Department of Experimental Pediatric Oncology, University Children's Hospital of Cologne, Medical

Faculty, Cologne, Germany.

\section{Dieter Beule}

$\mathrm{BIH}$

\section{David Torrents}

Barcelona Supercomputing Center

\section{Angelika Eggert}

Department of Pediatric Oncology and Hematology https://orcid.org/0000-0003-3476-8184

\section{Richard Koche}

MSKCC https://orcid.org/0000-0002-6820-5083

\section{Roland Schwarz}

Berlin Institute for Medical Systems Biology, Max Delbrück Center for Molecular Medicine in the Helmholtz Association

\section{Kerstin Haase}

Charite University Medicine https://orcid.org/0000-0002-0944-5618 


\section{Johannes Schulte}

Charité - Universitätsmedizin Berlin https://orcid.org/0000-0003-0671-1201

\section{Article}

\section{Keywords:}

Posted Date: March 7th, 2022

DOI: https://doi.org/10.21203/rs.3.rs-1396291/v1

License: (c) (1) This work is licensed under a Creative Commons Attribution 4.0 International License. Read Full License 
Elias Rodriguez-Fos ${ }^{1,2}$, Martin Burkert ${ }^{3}$, Montserrat Puiggròs ${ }^{4}$, Joern Toedling ${ }^{2}$, Nina Thiessen $^{5}$, Eric Blanc ${ }^{5}$, Annabell Szymansky ${ }^{2}$, Falk Hertwig ${ }^{2}$, Dieter Beule ${ }^{5}$, David Torrents ${ }^{4}$, Angelika Eggert ${ }^{1,2}$, Richard P. Koche ${ }^{6}$, Roland F. Schwarz ${ }^{3,7,8}$, Kerstin Haase ${ }^{1,9^{*}}$, Johannes H. Schulte $^{2 *}$, Anton G. Henssen ${ }^{1,2,3,5,9^{*}}$

${ }^{1}$ Experimental and Clinical Research Center (ECRC) of the MDC and Charité Berlin, Berlin, Germany.

$10{ }^{2}$ Department of Pediatric Oncology and Hematology, Charité - Universitätsmedizin Berlin, 11 corporate member of Freie Universität Berlin, Humboldt-Universität zu Berlin, Berlin,

12 Germany.

$13{ }^{3}$ Berlin Institute for Medical Systems Biology (BIMSB), Max Delbrück Center for Molecular 14 Medicine in the Helmholtz Association (MDC), Berlin, Germany

154 Barcelona Supercomputing Center, Joint Barcelona Supercomputing Center-Centre for

16 Genomic Regulation-Institute for Research in Biomedicine Research Program in

17 Computational Biology, Barcelona, Spain.

$18{ }^{5}$ Berlin Institute of Health, Berlin, Germany.

$19{ }^{6}$ Center for Epigenetics Research, Memorial Sloan Kettering Cancer Center, New York, NY, 20 USA

$21{ }^{7}$ Center for Integrated Oncology (CIO), Cancer Research Center Cologne Essen (CCCE),

22 Faculty of Medicine and University Hospital Cologne, University of Cologne, Cologne,

23 Germany

${ }^{8}$ BIFOLD - Berlin Institute for the Foundations of Learning and Data, Berlin, Germany

${ }^{9}$ German Cancer Consortium (DKTK), partner site Berlin, and German Cancer Research Center (DKFZ), Heidelberg, Germany.

*These authors jointly supervised this work

Correspondence should be addressed to: Henssenlab@gmail.com 


\section{Abstract}

33 Neuroblastoma is a pediatric solid tumor characterized by strong clinical heterogeneity. 34 Although certain complex genomic alterations, such as extrachromosomal DNA amplifications (ecDNA), have been recurrently detected in neuroblastomas, the mutational processes involved in their generation remain largely unclear. By examining the topography of complex rearrangements along with mutational signatures derived from all variant classes, we identify previously unrecognized co-occurring mutational footprints, which we termed mutational scenarios. We demonstrate that clinical neuroblastoma heterogeneity is linked to differences in the processes driving these mutational scenarios. Whereas high-risk $M Y C N$-amplified neuroblastoma genomes were characterized by signs of replication slippage and stress, homologous recombination-associated signatures defined high-risk non- $M Y C N$-amplified patients. Non-high-risk neuroblastomas, on the other hand, were marked by footprints of chromosome missegregation. This analysis provides a systematic perspective on the repertoire of mutational patterns that contribute to clinical neuroblastoma heterogeneity.

\section{Introduction}

The presence of somatic mutations is a hallmark of cancer genomes ${ }^{1}$. Somatic mutations present diverse typology and are a result of different endogenous and/or exogenous mutational processes, including replication errors, exposure to DNA damaging agents, expression of developmentally-restricted recombinases ${ }^{2}$, and errors in DNA repair mechanisms ${ }^{3}$. These processes imprint characteristic patterns of mutations in the genome, termed mutational signatures ${ }^{4}$. Recent analyses in multiple cancer types have extracted signatures associated with single-nucleotide variants $(\mathrm{SNVs})^{5,6}$, small insertions and deletions (indels) ${ }^{6}$, copy number alterations $(\mathrm{CNAs})^{7}$, and structural variants $(\mathrm{SVs})^{8}$. Some signatures are linked to known biological processes active in cancer, whereas others have yet unknown etiologies. In contrast to mutational signatures derived from SNVs, indels, and CNAs, the classification of SVs involving multiple genomic regions remains difficult. Various SV pattern classes have recently been defined based on their topography, e.g. extrachromosomal circular DNA ${ }^{9}$ (ecDNA), chromothripsis $^{10}$, chromoplexy ${ }^{11}$, templated insertion chains (TICs) ${ }^{12}$, breakage-fusion-bridge cycles $^{13}$ (BFB), complex non-cyclic amplicons ${ }^{14}(\mathrm{CnC})$, tyfona, rigma, and pyrgo ${ }^{15}$, amongst others. Even though the study of mutational signatures and structural variant patterns has advanced in recent years, it remains largely unclear how different mutational processes and variant topographies are mechanistically linked. 
Neuroblastoma is a pediatric solid tumor characterized by strong clinical heterogeneity ${ }^{16-18}$. Patients can be classified into different disease risk groups depending on their clinical, genetic, and molecular characteristics, namely low-, intermediate- or high-risk, with the latter presenting poor prognosis despite intensive therapy and surgery ${ }^{19}$. Previous reports focusing on SNV-related signatures have revealed signature SBS18, associated with reactive oxygen species, and signature SBS3, caused by impaired homologous recombination, to be the most prevalent in neuroblastoma ${ }^{20}$. Complex SV patterns as well as CNA signatures, on the other hand, have not yet been comprehensively investigated in neuroblastoma to date. Complex rearrangements involving oncogene amplicons are of particular importance in neuroblastoma ${ }^{21,22}$, as evidenced by the clinical impact of ecDNA-mediated oncogene amplification and its association with complex SV patterns. Recent studies have linked ecDNA to other complex variants such as chromothripsis and $\mathrm{BFB}^{23,24}$. However, our current understanding of complex rearrangement patterns and mutational processes, their cooccurrence, as well as their impact on clinical outcomes in neuroblastoma remains limited. Applying variant detection algorithms and signature analyses to Illumina short-read wholegenome sequencing (WGS) from a cohort of 114 neuroblastoma tumor-normal pairs, we extracted 12 catalogued mutational signatures associated with SNVs and indels as well as 19 recently defined CNA and SV signatures. We also identified 4 previously undescribed SV signatures, enabling a comprehensive description of the mutational processes active in neuroblastoma. Using statistical and graph-based methods, we integrated these mutational signatures with 8 complex SV pattern classes as well as clinical annotations resulting in a catalog of complex rearrangements in clinically heterogenous neuroblastomas.

\section{Results}

\section{Cohort characteristics}

90 To explore the topography of mutational processes in neuroblastoma, we analyzed WGS matched tumor-normal pairs from 114 patients. Tumors from all disease stages, sex, and age intervals were represented in our samples (Suppl. Fig. 1). 21\% of genomes were derived from high-risk $M Y C N$-amplified (HR MNA) neuroblastomas, 36\% from high-risk non- $M Y C N$ amplified cases (HR non-MNA), and 43\% from non-high-risk neuroblastomas (non-HR). We detected a median of 2,094 SNVs and indels per patient, 14 SVs per patient, and 19 CNAs per patient (Fig. 1). In line with previous reports ${ }^{25}$, high-risk non- $M Y C N$-amplified patients exhibited a significantly higher prevalence of all variant types (Fig. 1; Suppl. Fig. 2), with up to 1.8 and 6 times more somatic variants than $M Y C N$-amplified and non-high-risk patients, 
respectively. Pathognomonic chromosomal and driver gene alterations were identified at a similar prevalence in our cohort as described in other neuroblastoma cohorts ${ }^{26}$ (Fig. 1). Interestingly, high-risk non- $M Y C N$-amplified patients frequently harbored a $t(11 ; 17)$ translocation associated with gains of $17 \mathrm{q}$, losses of $11 \mathrm{q}$, or both ( $22 \%$ of samples), suggesting unbalanced translocations between chromosome 17 and 11 as the cause for 17q and 11q copy number alterations. $M Y C N$ amplifications detected in WGS were confirmed cytogenetically during routine clinical diagnostics using fluorescence in situ hybridization (FISH) and classified as either ecDNA or homogenous staining regions (Suppl. Table 3). Recurrently mutated genes included RAS pathway members (17.5\%, including $A L K)$, as well as PTPRD (12.3\%), TERT (8.8\%), SHANK2 (3.5\%), MDM2 (1.7\%), DAXX (0.9\%), and TP53 (0.9\%). In line with previous works ${ }^{27}, A T R X(7 \%)$, was exclusively altered in high-risk non-MYCNamplified patients. Apart from $A L K(8.8 \%)$, which was mainly affected by missense variants, most neuroblastoma driver genes were affected by SVs, highlighting the critical role of SVs in

SNV-based signatures SBS3, SBS18, and SBS40 are linked to different neuroblastoma risk groups

116 To provide a comprehensive overview of the mutational processes involved in neuroblastoma,

117 we first analyzed the SNV trinucleotide context from coding and non-coding regions of the 118 genome using mutSignatures ${ }^{28}$ (Fig. 2A,E; Suppl. Fig. 3; Suppl. Table 1). We recurrently identified six reference ${ }^{6}$ SNV-based signatures (SBS) in our cohort, indicating associated mutational processes are active in neuroblastoma. In line with previous reports ${ }^{20}, \mathrm{SBS} 3$ and SBS18, linked to defective homologous recombination repair (HRR) and reactive oxygen species (ROS), respectively, were recurrently identified in our neuroblastoma cohort (Fig. 2A; Suppl. Fig. 3A). Whereas SBS18, characterized by C $>$ A substitutions, was predominantly

124 observed in high-risk $M Y C N$-amplified patients $\left(\mathrm{P}<1.5 \times 10^{-4}\right), \mathrm{SBS} 3$, a flat signature, was observed to a significantly lower degree in that risk group $\left(\mathrm{P}<8.3 \times 10^{-6}\right)$ displaying a negative correlation with signature SBS18. Similarly to SBS3, SBS40, a clock-like signature associated with high patient age ${ }^{29}$, was highly anti-correlated with SBS18, inversely associated with clinical risk, and most prevalent in non-high-risk patients $\left(\mathrm{P}<2.4 \times 10^{-3}\right)$. Surprisingly, no association of SBS40 and age at diagnosis was observed, which may be due to overall young patient age in our cohort or could indicate that age-unrelated mutagenic processes are 
defective HRR and ROS are highly prevalent in neuroblastomas and differentially active across neuroblastoma risk groups.

Distinct indel-based signatures co-occur with SNV-based signatures SBS3, SBS18, and SBS40 and are associated with clinical risk groups

Next, we studied the mutational patterns of indels using YAPSA ${ }^{30}$. Considering the insertion and deletion lengths and genomic context, including repetitive sequences and microhomology, we identified six reference ${ }^{6}$ indel-based signatures (ID) in neuroblastoma genomes (Fig. 2B,E; Suppl. Fig. 4; Suppl. Table 1). ID1 and ID2, both characterized by 1 bp insertions and deletions at long thymine homopolymers attributed to replication slippage and found in most cancer entities $^{6}$, were also recurrently identified in our cohort. These signatures were significantly more frequent in patients with higher clinical risk, particularly in high-risk $M Y C N$-amplified patients $\left(\mathrm{P}<1.2 \times 10^{-4}\right)$. ID1 and ID2 positively correlated with SNV signature SBS18, which indicates co-occurrence of DNA damage by ROS and replication slippage in $M Y C N$-amplified samples. ID6, characterized by larger deletions ( $>5 \mathrm{bp}$ ) with larger microhomology, was also recurrently observed in our cohort. As expected, based on their common etiology associated with defective HRR, ID6 followed the same prevalence distribution between neuroblastoma risk groups as SBS3, being less predominant in high-risk $M Y C N$-amplified patients $(\mathrm{P}<2.9 \mathrm{x}$ $10^{-2}$ ) yet with preference for high-risk non- $M Y C N$-amplified neuroblastomas. Similarly, ID9, a signature of unknown etiology characterized by $1 \mathrm{bp}$ deletions at short cytosine and thymine homopolymers, was twice more frequent in high-risk non- $M Y C N$-amplified samples $(\mathrm{P}<1.5$ x $10^{-5}$ ). ID4, a signature enriched for deletions ( $>1 \mathrm{bp}$ ) at repeats and microhomology, recently associated with TOP1 mutational activity in cancer and healthy cells ${ }^{31}$, on the other hand, was significantly more prevalent in non-high-risk patients $\left(\mathrm{P}<1.3 \times 10^{-3}\right)$. ID4 displayed an association with SBS40 and high anticorrelation with all the other indel signatures, notably with the ones related to SBS3 (ID6, ID8, ID9) or SBS18 (ID1, ID2). This is in line with previous works in other cancer entities describing ID4 as mutually exclusive with signatures ID1 and ID2 ${ }^{6}$, and points to topoisomerases as a predominant source of mutagenesis in nonhigh-risk neuroblastoma. Thus, a subset of SNV-based and indel-based signatures co-occur in neuroblastomas from the same risk groups. 
Recent reports suggest that CNA patterns may allow the inference of signatures, offering more insights into molecular processes involved in their generation ${ }^{7,32}$. We measured the presence of 17 established CNA-associated signatures (CX, manuscript currently in revision) in our cohort of neuroblastomas (Fig. 2C,E; Suppl. Fig. 6; Suppl. Table 1). Signature differences are based on the number and size of copy number segments, and the difference in copy number between neighboring segments. Seven CX signatures significantly differed in frequency between risk groups. CX1, a signature associated with chromosomal arm changes potentially caused by chromosome missegregation and telomere disfunction, was the most prevalent CNA signature in our neuroblastoma cohort (Fig. 2C). It was associated with SBS3, although less prevalent in high-risk non- $M Y C N$-amplified patients $\left(\mathrm{P}<1.1 \times 10^{-2}\right) . \mathrm{CX} 3$, linked to defective HRR and replication stress-mediated long chromosomal segment changes, also co-occurred with SBS3 yet was significantly more frequent in high-risk non- $M Y C N$-amplified patients $(\mathrm{P}<$ $1.5 \times 10^{-2}$ ). CX1 and CX3 together accounted for more than $76 \%$ of the $\mathrm{CN}$ signatures' exposure identified in neuroblastomas, suggesting that neuroblastoma genomes are strongly shaped by chromosome missegregation and impaired HRR. Co-occurrence was observed between CX9, a signature attributed to replication stress-mediated focal amplifications, and SBS18, indicating ROS-induced replication stress as a potential cause of focal amplifications in neuroblastoma. CX14, characterized by footprints of chromosome missegregation via defective mitosis, was significantly associated with low-risk disease $\left(\mathrm{P}<8.2 \times 10^{-3}\right)$. CX5 , $\mathrm{CX} 7, \mathrm{CX} 9$, and CX11, on the other hand, were more frequent in high-risk $M Y C N$-amplified patients $\left(\mathrm{P}<3.2 \times 10^{-3}\right)$. Interestingly, these signatures are attributed to replication stress and were significantly associated with indel-based signatures ID1 and ID2, which are believed to result from replication slippage. Thus, CNA pattern analysis revealed that whereas $M Y C N$ driven neuroblastomas are characterized by replication stress and slippage, and high-risk non$M Y C N$-amplified neuroblastomas present higher prevalence of defective HRR, chromosome missegregation is a general feature of all neuroblastomas across risk groups.

\section{SV-based signatures reveal high prevalence of simple rearrangements associated with} transposase breakpoint signal sequence motifs in neuroblastoma

194 Recent reports suggest that site-specific recombinases may contribute to SV generation in embryonal tumors ${ }^{2}$. To evaluate the presence of such recurrent DNA sequences around SV breakpoints, we performed a de novo motif discovery analysis. Intriguingly, we recurrently

197 identified motifs closely resembling specific PGBD5 recombination signal sequences ${ }^{2}$ (Suppl.

198 Fig. 9), suggesting PGBD5 may contribute to structural variation in neuroblastoma. 
Next, we performed a de novo discovery of SV signatures (SV) based on the typology, size, and clustering of SVs in our neuroblastoma cohort using Palimpsest ${ }^{33}$ (Fig. 2D,E; Suppl. Fig. 5; Suppl. Table 1). After comparing these signatures to those identified in other tumor entities ${ }^{8}$, we defined four novel (SV3-6), presumably neuroblastoma-specific, and two known SV-based signatures (SV1-2), each differing with regards to their complexity, the enrichment in intraand interchromosomal rearrangements, and their size. SV1 was defined by complex intrachromosomal rearrangements, enriched in deletions, tandem-duplications, and inversions of sizes around 1-10 Mb. SV2 was characterized by complex intrachromosomal rearrangements larger than $10 \mathrm{Mb}$. SV1 and SV2 corresponded to reference SV-based signatures R6b and R6a, respectively, and were reported to be associated with MDM2 mutations and $C D K 4$ amplifications $^{8}$ (Suppl. Fig. 5C). SV3, on the other hand, a signature identified de-novo, was linked to simpler rearrangements and higher frequency of translocations in our cohort. Simple deletions smaller than $1 \mathrm{~kb}$ defined SV4. In SV5, complex rearrangements around $0.1-1 \mathrm{Mb}$ involving translocations were observed. High amounts of translocations together with simple duplications and deletions defined SV6, the most frequent

214 SV-associated signature in our cohort. In line with their complexity, SV signatures SV1, SV2, 215 and SV5 were positively associated with CN signature CX15, which is characterized by 216 clustered CNAs. Intriguingly, SV4, the second most prevalent SV signature in our cohort, 217 showed an inverse association with all the other SV signatures, notably with SV6, which may 218 indicate mutually exclusive underlying mutational processes as a cause of these variant 219 patterns. Even though no SV signatures significantly differed in frequency between risk groups, overall, signatures defined by simple rearrangements (SV3/SV4/SV6), were more frequent in neuroblastoma compared to those defined by complex SVs (SV1/SV2/SV5; $\left.\mathrm{P}<3.66 \times 10^{-5}\right)$. Thus, neuroblastomas are characterized by low-complexity SVs.

\section{Topography of complex structural rearrangements}

Recent reports have re-classified complex rearrangements topographically, considering both CNAs and SVs ${ }^{12,15,34}$. Even though some of these rearrangement patterns have been observed in neuroblastomas, e.g. ecDNA and chromothripsis ${ }^{21,25}$, the prevalence of most of the recently described patterns is currently unknown. To examine the topography of complex rearrangements in neuroblastoma, we used three complementary algorithms, JaBba ${ }^{15}$, Amplicon Architect ${ }^{35}$, and Shatterseek ${ }^{36}$ enabling the identification and reconstruction of eight complex variant classes: 
chromoplexy; (6) templated insertion chains (TIC), also described as chromoplexy-like; (7)

234 rigma, a cluster of simple deletions; (8) pyrgo, a cluster of tandem-duplications (Fig. 3,4A;

235 Suppl. Table 2). Interestingly, chromoplexy-like SVs were the most frequently observed SV

236 pattern in neuroblastomas and were detectable in 45 patients across all risk groups

237 (chromoplexy in 20 patients; TIC in 32 patients), suggesting a previously unknown

238 contribution to neuroblastoma genome remodeling. In line with our previous observations ${ }^{21}$,

239 ecDNA was also amongst the most frequent complex rearrangement classes in neuroblastomas

240 (34 patients). ecDNA was most prevalent in high-risk $M Y C N$-amplified patients $\left(\mathrm{P}<7.6 \times 10^{-}\right.$

$241^{8}$ ), in line with the fact that $M Y C N$ amplifications most often occur in the form of ecDNA ${ }^{21}$.

242 Most ecDNA-harboring tumors did not show signs of other known complex rearrangement

243 types (Fig. 4A). Only in a subset of cases ecDNA co-occurred with chromoplexy-like

244 rearrangements (TIC, chromoplexy), CnC, pyrgo, and BFB, suggesting ecDNA in these tumors

245 may either be involved in these complex rearrangements or have originated from them. In

246 contrast to previous reports ${ }^{23,24}$, no co-occurrence between ecDNA and chromothripsis was

247 observed in our cohort, indicating that chromothripsis may not contribute to ecDNA generation

248 in neuroblastoma to the same extent as in other tumor entities. Most neuroblastomas with signs

249 of BFB, on the other hand, also harbored ecDNAs, suggesting BFB as an important mechanism

250 of ecDNA generation and/or evolution in neuroblastoma. Overall, less than $1 \%$ of all SVs could

251 be assigned to previously defined complex variant patterns, with the highest fraction of SVs

252 attributed to $\mathrm{CnC}(0.98 \%$ of SVs), chromoplexy ( $0.40 \%$ of SVs), and ecDNA (0.20\%, Suppl.

253 Fig. 7), suggesting that most SVs either do not occur in recognizable patterns or that yet

254 unknown mutational patterns exist in neuroblastoma. Interestingly, complex SVs did not occur

255 uniformly throughout the genome (Fig. 4B). In line with our previous report showing ecDNA

256 as a hotspot for SVs, the MYCN locus on chromosome 2 was most recurrently affected by

257 complex variant patterns (19.3\% of patients), followed by the RASGRP3 locus, a Ras pathway

258 activator also located on chromosome 2, affected by complex rearrangements in $18.4 \%$ of

259 patients. Other previously unrecognized recurrently rearranged regions included cancer-related

260 loci such as SMARCE1 (6.1\% of patients), EBF1 (5.3\% of patients), a gene involved in

261 enhancer hijacking in neuroblastoma ${ }^{37}$, tumor suppressor $S D H A$ (5.3\% of patients), a known

262 hotspot for germline variants associated with neuroblastoma ${ }^{38}$, and the oncogene $H M G A 2$

263 (5.3\% of patients). Thus, topographically distinct complex variant patterns are more common

264 in neuroblastoma than previously anticipated and recurrently occur at sites of cancer-related

265 loci, suggesting their functional oncogenic importance. 

complex rearrangement patterns in neuroblastoma

269 The study of the interaction between complex variant patterns and mutational signatures within 270 different neuroblastoma risk groups suggests the existence of distinct co-occurring mutational 271 footprints with clinical relevance, termed mutational scenarios (Fig. 5,6A). The first mutational 272 scenario (scenario \#1), more frequent in high-risk $M Y C N$-amplified neuroblastomas, is defined 273 by footprints of DNA damage by ROS (SBS18), replication slippage (ID1/ID2), and replication stress (CX5/CX7/CX9/CX11). The presence of ecDNA was positively associated with scenario \#1 (Fig. 5A), designating ecDNA as a defining element of this mutational scenario and suggesting replication slippage/stress and ROS may contribute to ecDNA generation, in line with previous reports in mammalian cells ${ }^{39,40}$. Alternatively, the presence of ecDNA or high expression of oncogenes (e.g. $M Y C N$ ) encoded on them (Fig. 5B) may drive these mutational processes, in line with previous reports demonstrating that $M Y C$ can drive replication stress ${ }^{41}$.

280 The second mutational scenario (scenario \#2) is characterized by signs of impaired HRR (SBS3/ID6/CX3), single base deletions (ID9), and is associated with complex rearrangements such as chromoplexy, $\mathrm{CnC}$, and TIC. This mutational scenario is most frequent in high-risk non- $M Y C N$-amplified neuroblastomas. Interestingly, the presence of ecDNA was nearly mutually exclusive with defective homologous recombination-associated signatures defining scenario \#2 (Fig. 5A), implying that cells harboring ecDNA may require intact HRR. On the other hand, although not significantly associated with scenario \#2, chromothripsis displayed a unique correlation with homologous recombination-associated signatures (CX2), indicating that HRR may be involved in the repair of chromothriptic fragments, in line with previous reports in other tumor entities ${ }^{42}$. The third mutational scenario emerging from our analysis (scenario \#3) is linked to signatures of unknown etiology (SBS40), topoisomerase mutational activity (ID4), and signs of chromosome missegregation (CX14). This mutational scenario is highly prevalent in non-high-risk neuroblastomas. Thus, neuroblastomas harbor distinct combinations of mutational signatures and variant patterns, indicating that different mutational processes are active in each tumor.

\section{Mutational scenarios are linked to neuroblastoma clinical heterogeneity}

297 Previous reports in other tumor entities showed associations between complex rearrangements ${ }^{15}$, specific mutational processes, such as HRR deficiency ${ }^{43}$, and poor prognosis. Based on this, we hypothesized that neuroblastoma's clinical heterogeneity might

300 be in part explained by differences in complex rearrangement patterns and associated 
mutational processes, depicted by the three mutational scenarios described here. To test our hypothesis, we first evaluated the clinical impact of complex rearrangements in our cohort. Patients with tumors harboring ecDNA presented worse overall survival than patients with rearrangements affecting linear chromosomal DNA, such as chromoplexy, (Suppl. Fig. 8A,B; $\mathrm{P}<1 \times 10^{-4}$ by Log-rank test), suggesting that ecDNA supersedes other complex rearrangements in its association with adverse outcome. Subsequently, we assessed to what extent the three different mutational scenarios reflect neuroblastoma's clinical heterogeneity. We categorized each patient based on the presence of the set of signatures defining each mutational scenario 1-3, without considering their risk classification (Fig. 6; Suppl. Fig. 8).

310 Even though mutational scenarios are based on the co-ocurrence of mutational footprints within

311 risk groups, we observed that not all patients from a specific risk group were classified into the

312 same mutational scenario. Interestingly, patients from different mutational scenarios showed

313 pronounced differences in clinical outcome $\left(\mathrm{P}<1 \times 10^{-4}\right.$ by Log-rank test) confirming the 314 association of the defined scenarios with neuroblastoma's clinical heterogeneity. Moreover, a 315 subset of neuroblastomas harboring a mixture of signatures and patterns that could not be assigned to a single mutational scenario exhibited low overall survival, indicating that unknown mutational scenarios may exist. Thus, signatures defining mutational scenarios drive heterogeneous clinical outcomes in neuroblastoma.

\section{Discussion}

321 This study analyzed 114 neuroblastoma genomes and described the mutational processes and genomic rearrangement patterns in tumors from different clinical risk groups. Based on SNVs, indels, SVs, and CNAs patterns, we characterized three distinct mutational scenarios with clinical relevance. Scenario \#1 driven by DNA damage caused by ROS, replication slippage, and stress, most prevalent in high-risk $M Y C N$-amplified, ecDNA-harboring patients with low overall survival. Scenario \#2 driven by HRR-associated signatures and characterized by noncircular complex rearrangements, frequently observed in high-risk non- $M Y C N$-amplified patients with low survival. Finally, scenario \#3 characterized by footprints of chromosome missegregation, topoisomerase activity, and high presence of signature SBS40, prevalent in non-high-risk patients exhibiting good prognosis. Our findings demonstrate that clinical heterogeneity is accompanied by distinct mutational processes, raising the question whether these mutational processes contribute to the heterogeneous clinical outcomes.

333 Even though the identified mutational scenarios are linked to specific mutational processes, it 334 remains unknown what causes these processes to be active in neuroblastoma. Some molecular 
features in neuroblastomas have been linked to mutational processes in the past. For example, $M Y C$ can induce ROS, replication stress, and fork stalling ${ }^{41}$. It is tempting to speculate that MYCN could activate similar mutational processes, which would explain increased footprints of ROS, replication slippage, and stress observed in mutational scenario \#1, which was most prevalent in $M Y C N$-amplified patients. Further investigations using genetically controlled model systems may reveal such causal relationships between molecular cancer alterations and the presence of associated mutational signatures. Deficiencies in HRR can fuel the generation of simple and complex SVs ${ }^{44}$, such as BFB and chromothripsis $^{45}$. For example, mutations in $B R C A 1$ and $B R C A 2$ resulting in HRR deficiency are associated with high prevalence of certain SVs, previously termed $B R C A$ ness ${ }^{46}$. Although $B R C A 1 / 2$ mutations are not frequent in neuroblastoma ${ }^{47}$, we identified mutational signatures related to HRR deficiency in high-risk non- $M Y C N$-amplified patients. In line with our work, recent pediatric pan-cancer studies also observed signatures associated with HRR deficiency in neuroblastoma ${ }^{29}$. These reports, however, find lower exposures of these signatures in neuroblastomas. This could be due to differences in mutation calling sensitivity or signature misassigning. It remains unknown, which molecular alterations other than $B R C A 1 / 2$ deficiency could cause impaired HRR in neuroblastomas. Considering the high prevalence of these footprints in high-risk compared to non-high-risk patients, HRR deficiency may not only represent a mere feature of these tumors but could also contribute to their aggressive clinical behavior, similar as observed in HRR-deficient pancreatic cancer and gliomas ${ }^{48}$. Further investigations into the underlying causes of this mutational footprint in neuroblastoma may be especially relevant for future clinical trial designs, as HRR deficiency is associated with altered response to certain chemotherapeutic agents ${ }^{49}$, as well as increased sensitivity to pharmacological PARP trapping ${ }^{50}$. Thus, the identification of mutational patterns associated with HRR deficiency in high-risk neuroblastomas may have important implications for neuroblastoma risk and therapy stratification.

Mutational signature SBS40 is prevalent in different cancer types ${ }^{6}$, including neuroblastoma ${ }^{20}$. In line with previous reports ${ }^{29}$, we find high exposure of SBS40 in our neuroblastoma cohort within mutational scenario \#3. In contrast, we do not find SBS40 to be associated with signs of high genome instability such as chromothripsis in neuroblastoma, but find SBS40 to be most prevalent in non-high-risk patients exhibiting low numbers of structural rearrangements. This suggest that mutational processes responsible for SBS40 do not contribute to genome instability in neuroblastoma or that SBS40 is caused by different mutational processes in neuroblastoma than in other tumors. The latter could be explained by the fact that mutational 
signature analyses are a mathematical approximation of mutational processes. Thus, some processes may remain unidentified or even misassigned using current state-of-the-art algorithms $5,6,20,29$. This is especially interesting in the case of SBS40, which presents a mutational pattern with many similarities to other signatures, such as SBS5. For this reason, experimental systems validating causal relationships between signatures and molecular processes will be needed to help clarify the role of signatures exhibiting similar patterns.

In addition to the characterization of mutational processes through signature analyses, we provide the first complete catalog of recently described complex variant patterns ${ }^{15}$ in neuroblastoma, including chromothripsis, BFB, ecDNA, CnC, chromoplexy, TIC, and the novel rigma and pyrgo. Previous studies ${ }^{24,36}$ have reported a small subset of these complex rearrangement types in a few cancer-related loci such as $M Y C N, C D K 4$, and MDM2. We not only confirmed these rearrangements in our cohort, but also identified previously unidentified genomic hotspots of complex rearrangements, some of which were associated with cancerrelated genes such as RASGRP3, SMARCE1, EBF1, SDHA, and HMGA2. Moreover, we uncovered that many previously unrecognized complex rearrangements patterns recurrently affect cancer-related genes in neuroblastomas. This indicates that the functional contribution of these patterns is higher than anticipated and should be further explored.

ecDNA is one of the most prevalent genomic alterations in cancer ${ }^{51}$. We and others recently showed that it is not only a vehicle for oncogene amplification and high expression ${ }^{21}$, but also the most recurrent site of complex structural rearrangements ${ }^{14,21,22}$, indicating that ecDNA may serve as a substrate for genome remodeling. In line with this, footprints of BFB, chromothripsis and kataegis have been identified on ecDNA in several cancer entities ${ }^{23,24}$. We also observed signs of BFB on ecDNA in our neuroblastoma cohort, but did not observe footprints indicative of chromothripsis or APOBEC3-driven kataegis to the same extend as observed elsewhere ${ }^{52}$. This may be due to the fact that chromothripsis detection in highly amplified regions of the genome still remains challenging using currently available algorithms. However, even after manual curation of all ecDNA regions for the presence of non-canonical chromothripsis, we did not observe chromothriptic footprints, challenging the role of chromothripsis in the formation of ecDNA in neuroblastoma. Based on our observations, BFB may represent a more important process involved in ecDNA generation and/or structural evolution in neuroblastoma than previously estimated, indicating the need to further explore the relative contributions of complex rearrangement processes in the structural shaping of ecDNA.

In summary, our study provides a first comprehensive characterization of active mutational processes in neuroblastoma, offering new insights into the causes of genomic alterations 
involved in neuroblastoma genesis and progression. The three mutational scenarios presented here not only refine our understanding of neuroblastoma's clinical heterogeneity, but may also improve our understanding of how mutational processes contribute to the generation of different variant classes in cancer in general.

\section{Online Methods}

\section{Sequencing data}

This study is based on the analysis of 120 neuroblastoma primary tumor and matching control samples. Those samples are divided into two datasets. The first one, sequenced in Cologne, was completed in $2015^{37}$, and it is composed of WGS from 56 primary tumor and matching blood control samples (Illumina HiSeq). It was downloaded (https://www.ebi.ac.uk/ega/) under accession number EGAS00001001308 ${ }^{37}$. The second one, sequenced at the German Cancer Research Center, was completed in 2017-2019, and it includes WGS of 64 primary tumor and matching control samples (Illumina HiSeq X Ten). The quality of the raw data was assured using FastQC ${ }^{53}$. Reads were $3^{\prime}$ trimmed for both quality and adapter sequences, with reads removed if the length was shorter than 20 nucleotides. Burrows-Wheeler Aligner MEM ${ }^{54}$ v.0.7.15 with default parameters was used to align the reads to human reference assembly hg19. PCR and optical duplicates were marked with bammarkduplicates2 from BIOBAMBAM2 v2.0.87 (https://github.com/gt1/biobambam2).

Six patients have been excluded from the analyses due to an abnormally high number of mutations detected (CB2044, NBL47, NBL49, NBL50, NBL53, NBL54).

\section{Variant calling}

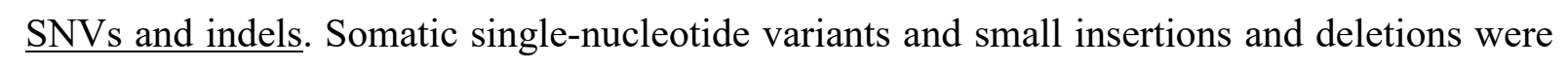
detected using Mutect2 v.4.1.8.1 from the GATK ${ }^{55}$ software package, with standard parameters according to GATK best practices recommendations. Variants were filtered using recommended filtering parameters, removing all germline mutations using a panel of normals.

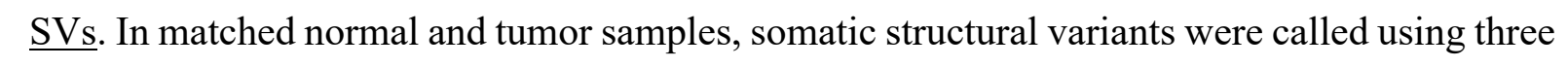
different variant callers: Delly2 ${ }^{56}$ v0.7.7, SvABA $^{57}$ v.1.1.0, and Novobreak ${ }^{58}$ v.1.1.3 with default parameters. To increase specificity without losing too much sensitivity, we intersected all the variants from the three callers and kept the ones detected at least in two of them. An exception has been made for insertions since we only identified them using SvABA. Variants with both breakpoints within a window of 500 bp were collapsed. Each variant included in the 
final set passed all the standard filters in at least one of the required two callers to be considered in our analysis.

438 CNAs. Allele-specific copy-number $(\mathrm{CN})$ profiles were generated from tumor and normal B 439 allele frequencies (BAFs) and LogR values using ASCAT $^{59}$ v2.6 with custom parameters. The 440 segmentation procedure from ASCAT was replaced with a custom implementation that only considers BAFs to determine start and end points of segments but still estimates the segment's coverage using the log coverage ratios. All $\mathrm{CN}$ profiles were inspected manually for quality control. For samples with an estimated tumor purity, below $60 \% \mathrm{CN}$ calling was rerun with adjusted purity and ploidy values that were manually selected after inspection of the sunrise plots and in agreement with pathology estimates of tumor purity. We assigned different copy number states to all profiles considering the local copy number and overall ploidy estimates. $\mathrm{CN}$ gain was defined as $\log 2((\mathrm{CNmajor}+\mathrm{CNminor}) /$ ploidy $)>0.3 ; \mathrm{CN}$ loss as $\log 2((\mathrm{CNmajor}$ + CNminor) / ploidy) $<-0.3$. CN amplifications and homozygous losses have been determined following COSMIC CNA parameters (https://cancer.sanger.ac.uk/cosmic/help/cnv/overview). In the case of amplifications, we added an additional filter of $\log 2((\mathrm{CNmajor}+\mathrm{CNminor}) /$ ploidy) $>1.25$ to increase specificity. For three patients no CNA results could be obtained (NBL31, NBL36, and NBL61).

\section{Identification of variants affecting genes and chromosome arms}

Chromosomal arms. Gains and losses of chromosomal arms have been determined intersecting the coordinates of the chromosomal regions with the CNA gains and losses, using bedtools v2.29.2 with parameters -wo -f 1 -F 0.60 (https://bedtools.readthedocs.io/). We considered an overlap of the whole CNA with at least $60 \%$ of the chromosomal arm.

459 Genes. We predicted the variant effect for SNV and indel calls using Ensembl VEP ${ }^{60}$ (Variant 460 Effect Predictor) v.102.0. Only SNVs and indels classified as missense and stop gained overlapping genes, were considered. For SV calls, we considered all the genes affected by SV breakpoints. We classified them in two types: (1) SV close to gene, defined as genes with at least one SV breakpoint at a distance of $+/-20 \mathrm{~kb}$; (2) SV within gene, defined as genes overlapping at least one SV breakpoint. For CNA calls, we considered the genes overlapping amplifications or homozygous deletions.

\section{Structural variant breakpoint analysis}

468 Base-pair accurate structural rearrangement calls from the merged structural variant set were considered for detailed breakpoint analysis. Using the hg19 reference genome, we obtained the 
sequence for a 50-bp window around each structural variant breakpoint. STREME v.5.3.0

471 (parameters -dna -nmotifs 15 -minw 5 -maxw 25 -objfun de) was used to identify motifs that were enriched with regard to a set of 1 million length-matched sequences randomly sampled from hg19. We compared those motifs with the previously published ${ }^{2}$ PGBD5-specific signal sequence. This analysis was done for all the samples together and also stratifying per risk groups.

\section{Mutational signatures analyses}

478 SNV signatures (SBS). To retrieve mutational signatures related to somatic single-nucleotide variants operating in neuroblastoma, we used the $\mathrm{R}$ package mutSignatures ${ }^{28}$ v.2.1.1. We started pre-processing all the SNVs in coding and non-coding regions detected in our patients. We applied a filtering step for non-SNV variants, followed by the tri-nucleotide context extraction for each of the variants which are classified in 96 different mutation types, or features, and counted across samples. Next, we ran the de-novo signature extraction step where non-negative matrix factorization (NMF) is performed. Complying with the pipeline recommendations, we computed 500 iterations, resulting in four de-novo signatures. Then, we evaluated which known COSMIC v.3.1 signatures (https://cancer.sanger.ac.uk/signatures/sbs/) matched our de-novo signatures, using a cosine distance threshold of $\leq 0.15$, obtaining six COSMIC signatures: SBS3, SBS12, SBS18, SBS36, SBS37, and SBS40. Finally, in the deconvolution step, we estimated known mutational signatures exposures in each patient and across neuroblastoma risk groups.

491 Indel signatures (ID). Mutational signatures associated with small somatic insertions and deletions $\left(<50 \mathrm{bp}\right.$ ) have been extracted using the R package YAPSA ${ }^{30}$ v.1.16.0, which includes PCAWG/COSMIC indel signatures (https://cancer.sanger.ac.uk/signatures/id/), following the standard pipeline. This pipeline starts with a pre-processing step of all the indels detected in our patients, in which we annotate the sequence context $10 \mathrm{bp}$ downstream and $60 \mathrm{bp}$ upstream of each variant, the variant type, and the length. In line with the SNV signature analysis, indels are classified in 83 different classes of features, and counted across samples. Next, we computed the supervised mutational signature analysis step, based on the linear combination decomposition function (LCD), obtaining the exposure for each known mutational signature in each patient. Signature exposure per neuroblastoma risk group was estimated from the exposure per patient. Recommended exposure cutoffs for each signature have been applied. 
502 From this analysis, we have obtained six PCAWG/COSMIC indel signatures: ID1, ID2, ID4, 503 ID6, ID8, and ID9.

504 CNA signatures (CX). To quantify the activity of copy number signatures in our samples, we used the 17 signatures that have been derived from over 6,000 tumors of the cancer genome atlas (https://www.cancer.gov/tcga) (manuscript in revision). Using the raw absolute copy number as obtained from ASCAT as input, first 43 features describing the fundamental features of the copy number profiles are extracted. Afterwards the exposure for each previously described signature in each of our samples is calculated based on the posterior distribution of the extracted features.

511 SV signatures (SV). Mutational signatures associated with somatic structural variants have 512 been extracted using the R package Palimpsest ${ }^{33}$ v.2.0.0 following the standard pipeline for 513 this variant type. This pipeline starts with a pre-processing step in which we annotate the type 514 (deletions, inversions, tandem-duplications, and translocations), size ( $<1 \mathrm{~kb}, 1-10 \mathrm{~kb}, 10-100$

$515 \mathrm{~kb}, 100 \mathrm{~kb}-1 \mathrm{Mb}, 1-10 \mathrm{Mb}$, and $>10 \mathrm{Mb}$ ), and clustered nature of rearrangements (distinguish between clustered and non-clustered events). SVs are classified into 38 different classes of features, and counted across samples. Next, we computed the de-novo signature extraction step, 518 based on NMF. As a result, we obtained six de-novo signatures that were named based on the 519 prevalence of SVs in the different features classes: (1) SV1, complex intrachromosomal SVs; (2) SV2, large complex intrachromosomal SVs;

(3) SV3, large simple intra and interchromosomal SVs;

(4) SV4, small deletions;

(5) SV5, complex intra and interchromosomal SVs; (6) SV6, simple duplications, deletions, and interchromosomal SVs. In the final step of the pipeline, we estimated the exposure of each signature in each sample, and across neuroblastoma risk groups. In order to evaluate if our 6 de-novo SV-based signatures found in neuroblastoma matched any of the 21 reference SV signatures from previous pancancer studies ${ }^{8}$ we used the deconvolution_compare function of Palimpsest obtaining cosine similarities between signatures. Using the same cosine distance threshold for the SNV analysis

$528(\leq 0.15)$ we identified two matches. De-novo signatures SV1 and SV2, corresponded to reference signatures $\mathrm{R} 6 \mathrm{~b}$ and $\mathrm{R} 6 \mathrm{a}^{8}$.

530 From the patient's exposure, we assessed the contribution of each of the different mutational signatures within neuroblastoma risk groups.

\section{Complex rearrangements calling and classification}


534 To detect and reconstruct all linear and circular complex rearrangements types in neuroblastoma, we used three complementary algorithms: JaBba ${ }^{15}$ v1.0, Amplicon Architect ${ }^{35}$ v.1.2, and the R package Shatterseek ${ }^{36}$ v.0.5.

537 JaBba. JaBba has been run using standard parameters and following the best practices pipeline, 538 including the pre-processing steps using fragCounter and dryclean to correct for GC content and mappability and denoise the coverage data using a panel of normals. From these steps, we obtained the coverage input that we use along our SV calls to run the pipeline. Then, following what has been done in previous studies ${ }^{15}$, the output from JaBba was run into gGnome v.0.1 to classify the different complex SVs into rigma, pyrgo, TIC, chromoplexy, chromothripsis, BFB, double minute (DM), and tyfonas. gGnome was also used to generate the figures with the reconstruction of the rearrangements.

545 Amplicon Architect. We used the copy number profiles obtained from ASCAT and the bam files of all tumor samples as input to PrepareAA ${ }^{35}$ (https://github.com/jluebeck/PrepareAA), a wrapper function that handles the preprocessing and execution of AmpliconArchitect v.1.2. We predicted the types of amplifications present in Amplicon Architect's ${ }^{35}$ output using AmpliconClassifier ${ }^{14}$ v.0.4.6 with standard parameters except for - force=TRUE. We classified circular amplicons in ecDNA and BFB, and linear amplicons in complex non-cyclic amplicons $(\mathrm{CnC})$.

552 ShatterSeek. ShatterSeek ${ }^{36}$ has been run from CNA and SV calls using standard parameters to detect chromothripsis. The filtering steps presented in the tutorial to obtain high confidence calls have been followed: at least 6 interleaved intrachromosomal SVs, 7 contiguous segments oscillating between $2 \mathrm{CN}$ states, significant fragment join tests, and either significant chromosomal enrichment or exponential distribution of breakpoints test. In order to include chromothriptic candidates in our results that might affect highly amplified regions, we also called low-confidence chromothripsis having at least 6 interleaved intrachromosomal SVs and 7 contiguous segments. In these cases, a final step of visual inspection was performed. The three complex rearrangement calling pipelines have been run using the hg19 reference genome. To gain sensitivity in the reconstruction of the complex SVs, we used the SVs that passed all the standard variant calling filters in at least one caller. Complex rearrangements detected by at least one method were considered. All complex rearrangements detected in the same patient overlapping the same genomic region between methods have been merged, keeping the most informative classification. 
568 Co-occurrence of the different complex rearrangement types was computed considering the 569 presence of the different rearrangement classes within the same patient. The figure has been 570 created using the R package ComplexUpset v.1.2.1 (https://github.com/krassowski/complex571 upset).

572 The ratio of SVs involved in complex rearrangements has been extracted establishing the ratio

573 between the number of SVs junctions from those rearrangements and the total number of SVs 574 in each patient.

575 For each patient, we obtained the breakpoint coordinates of each of the complex SVs. Then, 576 splitting the genome into $1 \mathrm{Mb}$ regions, we extracted which genomic regions are affected by 577 complex rearrangements in our cohort. This analysis has been done for the different 578 neuroblastoma risk groups. Regions encompassing neuroblastoma relevant cancer-related 579 genes, from the COSMIC cancer gene census, affected in $>5 \%$ of the samples from our whole 580 cohort have been highlighted in the figure.

581

$582 \quad$ Kataegis analysis

583 We consider a cluster of kataegis when we called 6 or most consecutive SNVs with an 584 intermutation distance of $<1 \mathrm{~kb}$. The same method has been used in previous studies ${ }^{23}$.

\section{Mutational scenarios analysis}

587 For the definition of the three different mutational scenarios, we considered all the biological 588 features identified in this work such as mutational signatures, their associated mutational processes, complex rearrangement classes, and risk group classification. Only biological features significantly present/correlated within a risk group are considered defining the mutational scenario associated to that risk group. The significance level has been established at $\mathrm{P}<0.05$. We established a Spearman correlation threshold of $0.3 /-0.3$ to consider that the feature of interest has a moderate/strong relationship with the features characterizing the mutational scenario and, as a result, can be defining. If a signature or complex rearrangement was equally distributed across two or more risk groups, significantly correlated with signatures or mutational processes from two or more risk groups, or did not reach the Spearman correlation threshold, then, it was not considered as a feature with enough clinical relevance to stratify between patients and as a result was excluded from the final characterization of the mutational scenarios.

600 
602 All comparisons between distributions in the different neuroblastoma risk groups were made 603 using the non-parametric Wilcoxon rank-sum test. To assess if there are differences between 604 risk groups, we used the non-parametric Kruskal-Wallis test. The relationship between different variables such as signatures, complex rearrangements, and mutated genes has been calculated using Spearman's correlation coefficient. All statistical analyses have been corrected by multiple testing when applicable using the false discovery rate (FDR) correction. All pvalues in the main text have been obtained using the Wilcoxon rank-sum test unless stated otherwise. The significance level has been established at $\mathrm{P}<0.05$.

610 Log-rank tests were used for survival analysis across subgroups. To assess the clinical impact 611 of the three defined mutational scenarios, we stratified our patients by presence/absence of the

612 whole set of mutational signatures and complex rearrangements that define each scenario 613 without considering their neuroblastoma risk classification. Patients presenting a mixture of 614 signatures and patterns that could not be assigned to a single mutational scenario were 615 classified as Mix of signatures. The subgroups for the analysis were: Mutational scenario \#1 (n $616=7)$, Mutational scenario \#2 $(n=14)$, Mutational scenario \#3 $(n=30)$, and Mix of signatures $617(\mathrm{n}=56)$. To assess the clinical impact of complex rearrangements in this tumor, we stratified 618 our patients by the presence/absence of linear and circular complex SVs. The subgroups for 619 the analysis were: Linear comp. rearrang. - / ecDNA - $(\mathrm{n}=35)$, Linear comp. rearrang. $+/$ 620 ecDNA - $(\mathrm{n}=45)$, Linear comp. rearrang. - / ecDNA + $(\mathrm{n}=16)$, and Linear comp. rearrang. + $621 / \mathrm{ecDNA}+(\mathrm{n}=18)$.

622

\section{Patient samples and clinical data access}

624 This study comprised the analyses of tumor and blood samples of patients diagnosed with neuroblastoma between 1991 and 2016. Patients were registered and treated according to the trial protocols of the German Society of Pediatric Oncology and Hematology (GPOH). This study was conducted in accordance with the World Medical Association Declaration of Helsinki (2013) and good clinical practice; informed consent was obtained from all patients or their guardians. The collection and use of patient specimens was approved by the institutional review boards of Charité-Universitätsmedizin Berlin and the Medical Faculty, University of Cologne. Specimens and clinical data were archived and made available by CharitéUniversitätsmedizin Berlin or the National Neuroblastoma Biobank and Neuroblastoma Trial Registry (University Children's Hospital Cologne) of the GPOH. The MYCN gene copy number was determined as a routine diagnostic method using fluorescence in situ hybridization (FISH). Results of the FISH analysis have been confirmed by quantitative polymerase chain 
reaction (qPCR). DNA and total RNA were isolated from tumor samples with at least $60 \%$

\section{References}

640 1. Greenman, C. et al. Patterns of somatic mutation in human cancer genomes. Nature 446, 641 153-158 (2007).

642 2. Henssen, A. G. et al. PGBD5 promotes site-specific oncogenic mutations in human tumors. Nat Genet 49, 1005-1014 (2017).

3. Jeggo, P. A., Pearl, L. H. \& Carr, A. M. DNA repair, genome stability and cancer: a historical perspective. Nat Rev Cancer 16, 35-42 (2016).

4. Koh, G., Degasperi, A., Zou, X., Momen, S. \& Nik-Zainal, S. Mutational signatures: emerging concepts, caveats and clinical applications. Nat Rev Cancer 21, 619-637 (2021).

5. Australian Pancreatic Cancer Genome Initiative et al. Signatures of mutational processes in human cancer. Nature 500, 415-421 (2013).

6. PCAWG Mutational Signatures Working Group et al. The repertoire of mutational signatures in human cancer. Nature 578, 94-101 (2020).

7. Macintyre, G. et al. Copy number signatures and mutational processes in ovarian carcinoma. Nat Genet 50, 1262-1270 (2018).

8. Degasperi, A. et al. A practical framework and online tool for mutational signature analyses show inter-tissue variation and driver dependencies. Nat Cancer 1, 249-263 (2020).

657

9. Turner, K. M. et al. Extrachromosomal oncogene amplification drives tumour evolution and genetic heterogeneity. Nature 543, 122-125 (2017).

659 10. Stephens, P. J. et al. Massive Genomic Rearrangement Acquired in a Single

660 Catastrophic Event during Cancer Development. Cell 144, 27-40 (2011).

661 11. Baca, S. C. et al. Punctuated evolution of prostate cancer genomes. Cell 153, 666-677 662 (2013).

663 12. Li, Y. et al. Patterns of somatic structural variation in human cancer genomes. Nature 578, 112-121 (2020).

665 13. Zakov, S., Kinsella, M. \& Bafna, V. An algorithmic approach for breakage-fusion666 bridge detection in tumor genomes. Proceedings of the National Academy of Sciences 110, 667 5546-5551 (2013).

668 14. Kim, H. et al. Extrachromosomal DNA is associated with oncogene amplification and 669 poor outcome across multiple cancers. Nat Genet 52, 891-897 (2020). 
670

671

672

673

674

675

676

677

678

679

680

681

682

683

684

685

686

687

688

689

690

691

692

693

694

695

696

697

698

699

700

701

702

15. Hadi, K. et al. Distinct Classes of Complex Structural Variation Uncovered across Thousands of Cancer Genome Graphs. Cell 183, 197-210.e32 (2020).

16. Bown, N. Neuroblastoma tumour genetics: clinical and biological aspects. Journal of Clinical Pathology 54, 897-910 (2001).

17. Brodeur, G. M. Neuroblastoma: biological insights into a clinical enigma. Nat Rev Cancer 3, 203-216 (2003).

18. Capasso, M. \& Diskin, S. J. Genetics and Genomics of Neuroblastoma. in Cancer Genetics (ed. Pasche, B.) vol. 155 65-84 (Springer US, 2010).

19. Cohn, S. L. et al. The International Neuroblastoma Risk Group (INRG) classification system: an INRG Task Force report. J Clin Oncol 27, 289-297 (2009).

20. Brady, S. W. et al. Pan-neuroblastoma analysis reveals age- and signature-associated driver alterations. Nat Commun 11, 5183 (2020).

21. Koche, R. P. et al. Extrachromosomal circular DNA drives oncogenic genome remodeling in neuroblastoma. Nat Genet 52, 29-34 (2020).

22. Helmsauer, K. et al. Enhancer hijacking determines extrachromosomal circular MYCN amplicon architecture in neuroblastoma. Nat Commun 11, 5823 (2020).

23. Shoshani, O. et al. Chromothripsis drives the evolution of gene amplification in cancer. Nature 591, 137-141 (2021).

24. Rosswog, C. et al. Chromothripsis followed by circular recombination drives oncogene amplification in human cancer. Nat Genet (2021) doi:10.1038/s41588-02100951-7.

25. Molenaar, J. J. et al. Sequencing of neuroblastoma identifies chromothripsis and defects in neuritogenesis genes. Nature 483, 589-593 (2012).

26. Pugh, T. J. et al. The genetic landscape of high-risk neuroblastoma. Nat Genet $\mathbf{4 5}$, 279-284 (2013).

27. Zeineldin, M. et al. MYCN amplification and ATRX mutations are incompatible in neuroblastoma. Nat Commun 11, 913 (2020).

28. Fantini, D., Vidimar, V., Yu, Y., Condello, S. \& Meeks, J. J. MutSignatures: an R package for extraction and analysis of cancer mutational signatures. Sci Rep 10, 18217 (2020).

29. Thatikonda, V. et al. Comprehensive analysis of mutational signatures in pediatric cancers. http://biorxiv.org/lookup/doi/10.1101/2021.09.28.462210 (2021) doi:10.1101/2021.09.28.462210. 
30. Daniel Huebschmann, Z. G. YAPSA. (Bioconductor, 2017). doi:10.18129/B9.BIOC.YAPSA.

31. Reijns, M. A. M. et al. Signatures of TOP1 transcription-associated mutagenesis in cancer and germline. Nature (2022) doi:10.1038/s41586-022-04403-y.

32. Steele, C. D. et al. Undifferentiated Sarcomas Develop through Distinct Evolutionary Pathways. Cancer Cell 35, 441-456.e8 (2019).

33. Letouzé, E. et al. Mutational signatures reveal the dynamic interplay of risk factors and cellular processes during liver tumorigenesis. Nat Commun 8, 1315 (2017).

34. Campbell, P. J. et al. The patterns and dynamics of genomic instability in metastatic pancreatic cancer. Nature 467, 1109-1113 (2010).

35. Deshpande, V. et al. Exploring the landscape of focal amplifications in cancer using AmpliconArchitect. Nat Commun 10, 392 (2019).

36. Cortés-Ciriano, I. et al. Comprehensive analysis of chromothripsis in 2,658 human cancers using whole-genome sequencing. Nat Genet 52, 331-341 (2020).

37. Peifer, M. et al. Telomerase activation by genomic rearrangements in high-risk neuroblastoma. Nature 526, 700-704 (2015).

38. Dubard Gault, M. et al. Germline SDHA mutations in children and adults with cancer. Cold Spring Harb Mol Case Stud 4, a002584 (2018).

39. Paulsen, T., Kumar, P., Koseoglu, M. M. \& Dutta, A. Discoveries of Extrachromosomal Circles of DNA in Normal and Tumor Cells. Trends in Genetics 34, 270-278 (2018).

40. Dillon, L. W. et al. Production of Extrachromosomal MicroDNAs Is Linked to Mismatch Repair Pathways and Transcriptional Activity. Cell Reports 11, 1749-1759 (2015).

41. Rohban, S. \& Campaner, S. Myc induced replicative stress response: How to cope with it and exploit it. Biochimica et Biophysica Acta (BBA) - Gene Regulatory Mechanisms 1849, 517-524 (2015).

42. Voronina, N. et al. The landscape of chromothripsis across adult cancer types. Nat Commun 11, 2320 (2020).

43. Dai, W. et al. Clinical Outcome-Related Mutational Signatures Identified by Integrative Genomic Analysis in Nasopharyngeal Carcinoma. Clin Cancer Res 26, 64946504 (2020).

44. Currall, B. B., Chiangmai, C., Talkowski, M. E. \& Morton, C. C. Mechanisms for Structural Variation in the Human Genome. Curr Genet Med Rep 1, 81-90 (2013). 
45. Piazza, A. \& Heyer, W.-D. Homologous Recombination and the Formation of Complex Genomic Rearrangements. Trends in Cell Biology 29, 135-149 (2019).

46. Byrum, A. K., Vindigni, A. \& Mosammaparast, N. Defining and Modulating 'BRCAness'. Trends in Cell Biology 29, 740-751 (2019).

47. Mersch, J. et al. Cancers associated with BRCA 1 and BRCA 2 mutations other than breast and ovarian: BRCA and Other Cancers. Cancer 121, 269-275 (2015).

48. Heeke, A. L. et al. Prevalence of Homologous Recombination-Related Gene Mutations Across Multiple Cancer Types. JCO Precision Oncology 1-13 (2018) doi:10.1200/PO.17.00286.

49. Zhang, J. The role of BRCA1 in homologous recombination repair in response to replication stress: significance in tumorigenesis and cancer therapy. Cell Biosci 3, 11 (2013).

50. Creeden, J. F. et al. Homologous recombination proficiency in ovarian and breast cancer patients. BMC Cancer 21, 1154 (2021).

51. Verhaak, R. G. W., Bafna, V. \& Mischel, P. S. Extrachromosomal oncogene amplification in tumour pathogenesis and evolution. Nat Rev Cancer 19, 283-288 (2019).

52. Bergstrom, E. N. et al. Mapping clustered mutations in cancer reveals APOBEC3 mutagenesis of ecDNA. Nature (2022) doi:10.1038/s41586-022-04398-6.

53. FastQC. (2015).

54. Li, H. Aligning sequence reads, clone sequences and assembly contigs with BWAMEM. arXiv:1303.3997 [q-bio] (2013).

55. McKenna, A. et al. The Genome Analysis Toolkit: A MapReduce framework for analyzing next-generation DNA sequencing data. Genome Res. 20, 1297-1303 (2010).

56. Rausch, T. et al. DELLY: structural variant discovery by integrated paired-end and split-read analysis. Bioinformatics 28, i333-i339 (2012).

57. Wala, J. A. et al. SvABA: genome-wide detection of structural variants and indels by local assembly. Genome Res. 28, 581-591 (2018).

58. Chong, Z. et al. novoBreak: local assembly for breakpoint detection in cancer genomes. Nat Methods 14, 65-67 (2017).

59. Van Loo, P. et al. Allele-specific copy number analysis of tumors. Proceedings of the National Academy of Sciences 107, 16910-16915 (2010).

60. McLaren, W. et al. The Ensembl Variant Effect Predictor. Genome Biol 17, 122 (2016). 
772 We thank F. Dubois and O. Shoshani for critical discussions. A.G.H. is a participant in the 773 Berlin Institute of Health-Charité Clinical Scientist Program funded by the Charité774 Universitätsmedizin Berlin and the Berlin Institute of Health. A.G.H. is supported by the 775 Deutsche Forschungsgemeinschaft (DFG, German Research Foundation, 398299703) and the 776 European Research Council (ERC) under the European Union's Horizon 2020 research and 777 innovation program (grant agreement No. 949172). J.H.S. and K.H. are supported by the 778 German Cancer Consortium (DKTK). E.R-F. is supported by the Alexander von Humboldt 779 Foundation. This project was supported by the Berlin Institute of Health within the 780 collaborative research project TERMINATE-NB (CRG04). We thank the patients and their 781 parents for granting access to the tumor specimens and clinical information that were analyzed 782 in this study. We thank B. Hero, H. Düren and N. Hemstedt of the Neuroblastoma Biobank and 783 Neuroblastoma Trial Registry (University Children's Hospital Cologne) of the GPOH for 784 providing samples and clinical data. Computation was performed on the HPC for Research 785 cluster of the Berlin Institute of Health and the MareNostrum of the Barcelona Supercomputing 786 Center. 


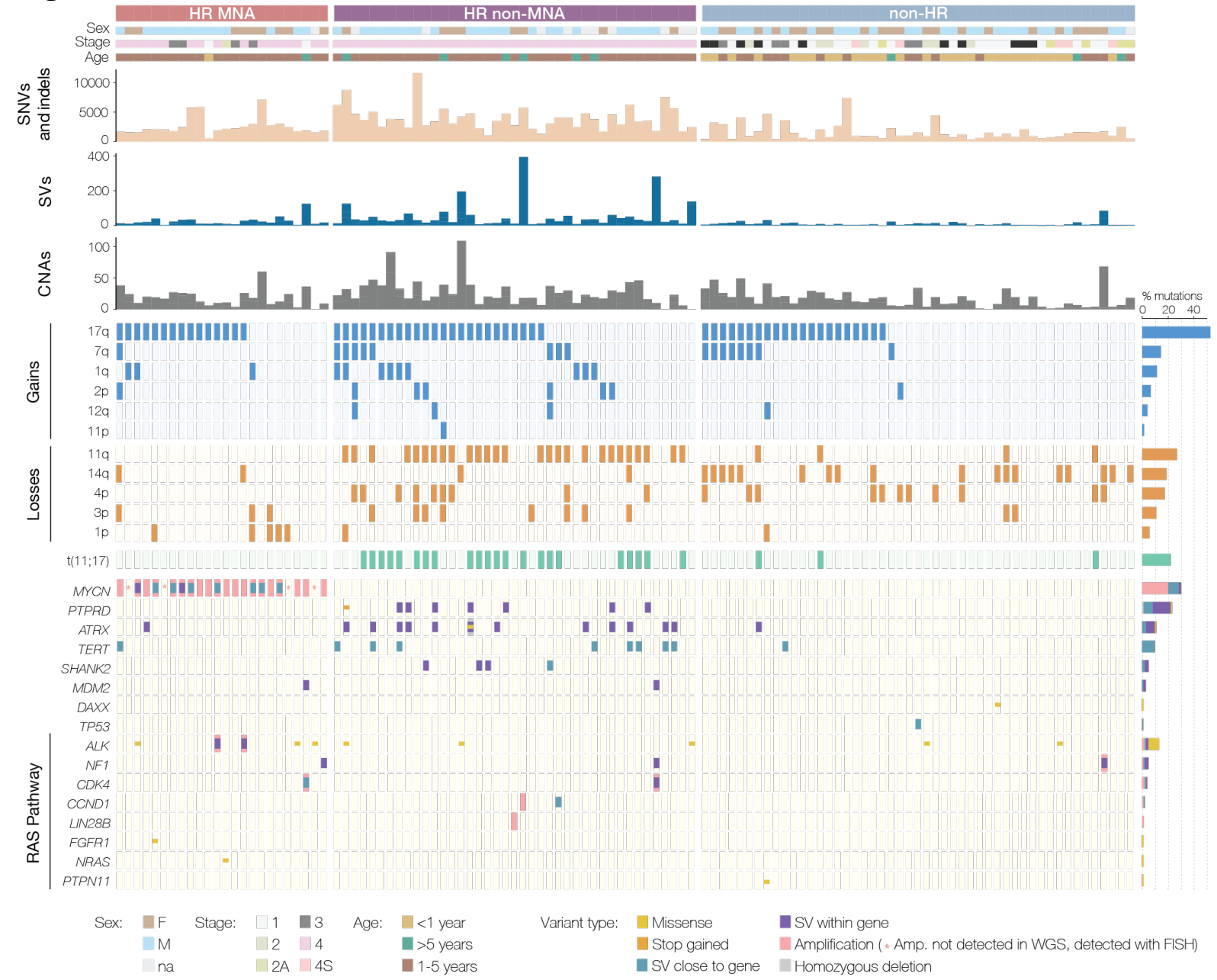

Figure 1. Landscape of somatic variants in clinically heterogenous neuroblastomas. Top, risk group (HR MNA: high-risk $M Y C N$-amplified, $\mathrm{n}=24$; HR non-MNA: high-risk non$M Y C N$-amplified, $\mathrm{n}=41$; non-HR: non-high-risk, $\mathrm{n}=49$ ), sex, stage of the disease, and age. Next, number of SNVs and indels, SVs, and CNAs in each of the 114 neuroblastoma patients. Middle, presence of known neuroblastoma chromosomal arm gains (blue), losses (orange), and translocations between chromosome 11 and 17 (green) in each patient. Bottom, presence of mutations in known neuroblastoma driver genes, including genes of the RAS pathway in each patient. Genes are ranked based on their mutation frequency in the cohort. The variant types considered here are SNVs and indels classified as missense and stop gained variants, SVs close and within the gene, amplifications, and homozygous deletions. Color indicates the type of mutation (key at the bottom). Patients with more than one type of variant in a gene have multiple colors designated. The right histogram indicates the percentage of mutations affecting each genomic feature in the whole cohort. Variant type is indicated by color. 
a
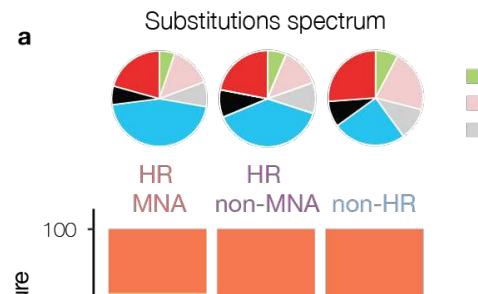

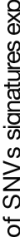

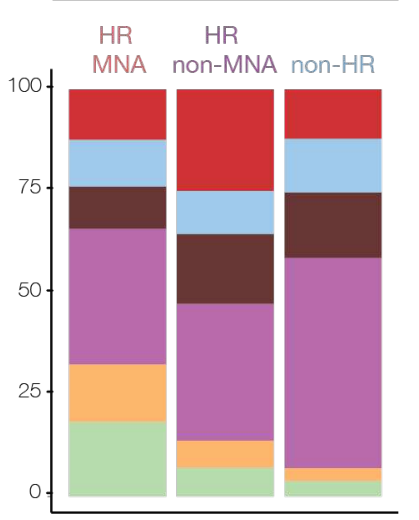

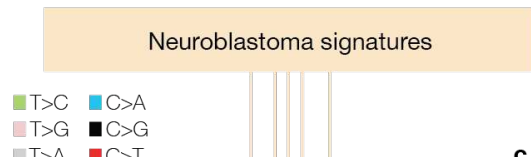

c
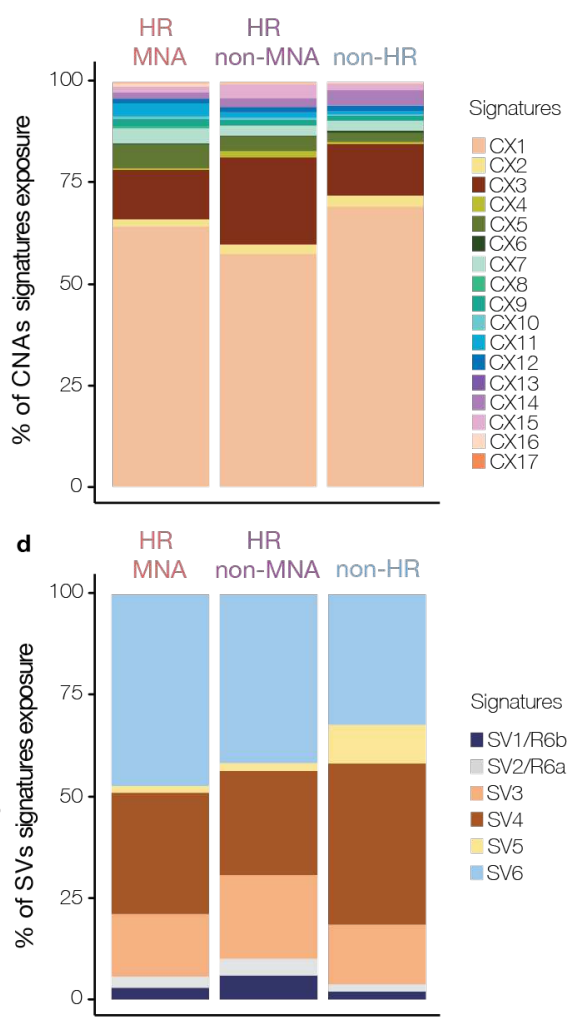
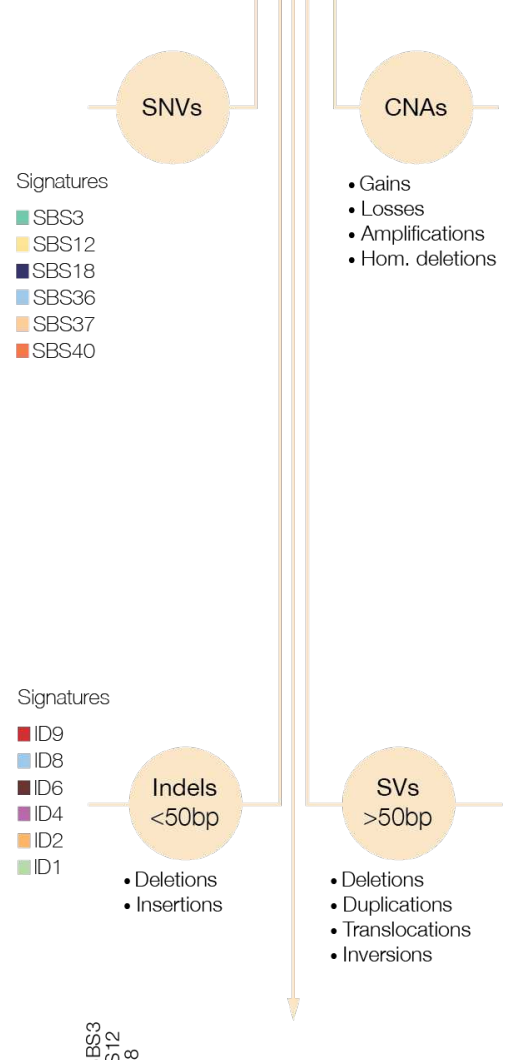

- Inversions

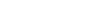

Correlation

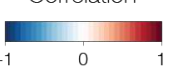

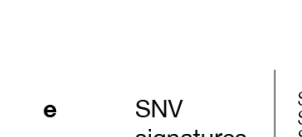
neuroblastoma clinical risk groups. (a) Top, spectrum of SNVs in the different risk groups. Bottom, exposure (in percentage) of the six SNV-associated signatures (SBS) identified in our neuroblastoma cohort by clinical risk group. Each color displays a different signature: SBS3, SBS12, SBS18, SBS36, SBS37, SBS40. (b) Exposure (in percentage) of the six indel signatures (ID; insertions and deletions $<50 \mathrm{bp}$ ) identified in our cohort by clinical risk group. Each color displays a different signature: ID1, ID2, ID4, ID6, ID8, ID9. (c) Exposure (in percentage) of the 17 CNA signatures (CX; gains, losses, amplifications, and homozygous deletions) extracted in our cohort by clinical risk group. Each color displays a different signature: CX1-CX17. (d) 
839 and inversions) identified in our cohort by clinical risk group. Each color displays a different 840 signature: SV1-SV6. SV1 and SV2 correspond to reference signatures R6b and R6a, 841 respectively. In a-d, columns are ordered by neuroblastoma clinical risk classification. (e) 842 Heatmap depicting the positive (red) and negative (blue) correlations between the signatures 843 associated with different types of mutations (SNV, indel, SV, and CNA). Colors display the 844 Spearman correlation coefficient. Only significant correlations are included $(\mathrm{P}<0.05$, FDR 845 correction). 


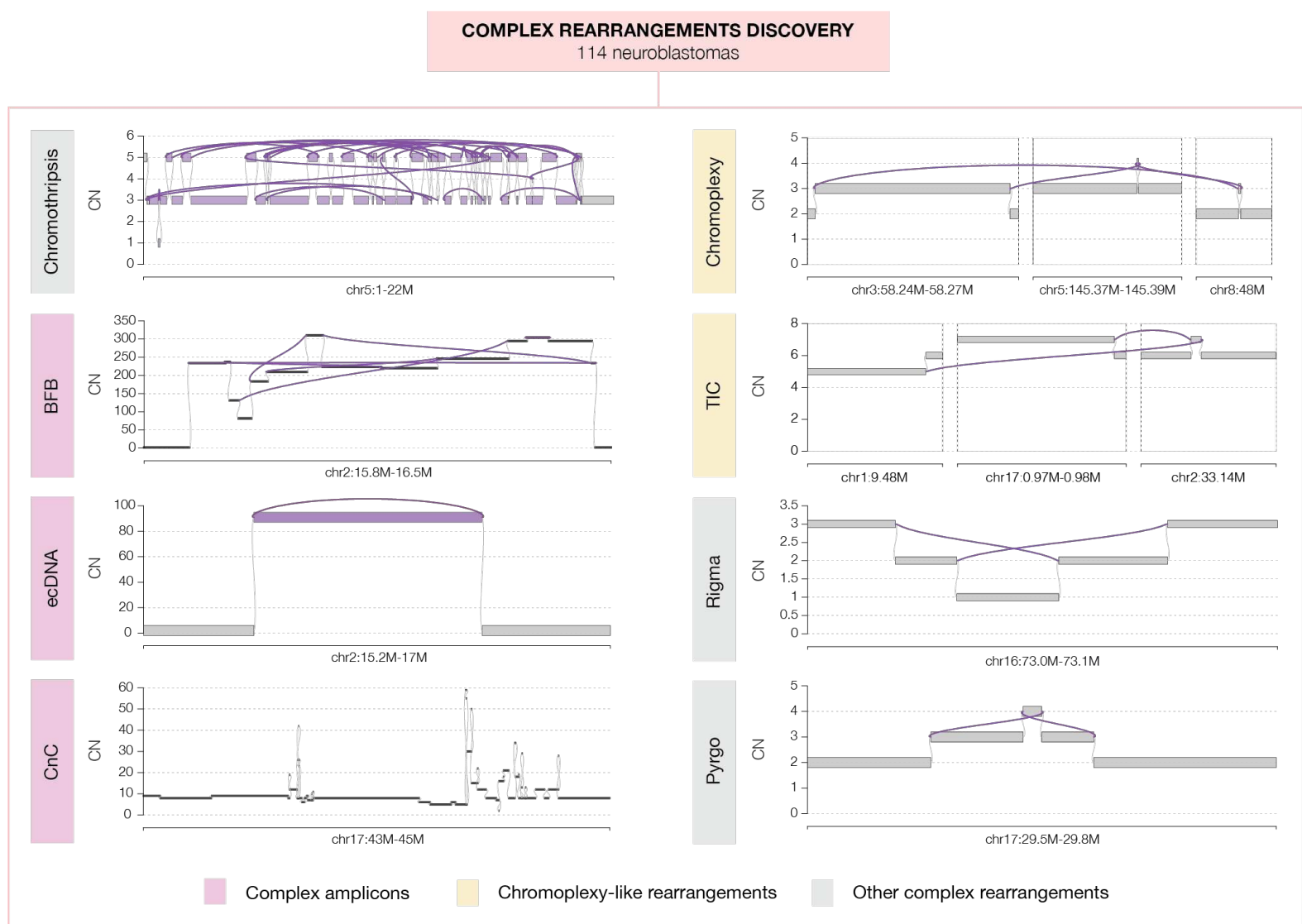

Figure 3. Different classes of complex rearrangement patterns are detected in neuroblastoma. Eight complex rearrangement types identified in our cohort of 114 neuroblastoma patients: chromothripsis, breakage-fusion-bridge cycles (BFB), extrachromosomal circular DNA (ecDNA), complex non-cyclic amplicons (CnC), chromoplexy, templated insertion chains (TIC), rigma (cluster of deletions), and pyrgo (cluster of tandem-duplications). Colors indicate the class of rearrangement: complex amplicons, chromoplexy-like, and others (key at the bottom). 

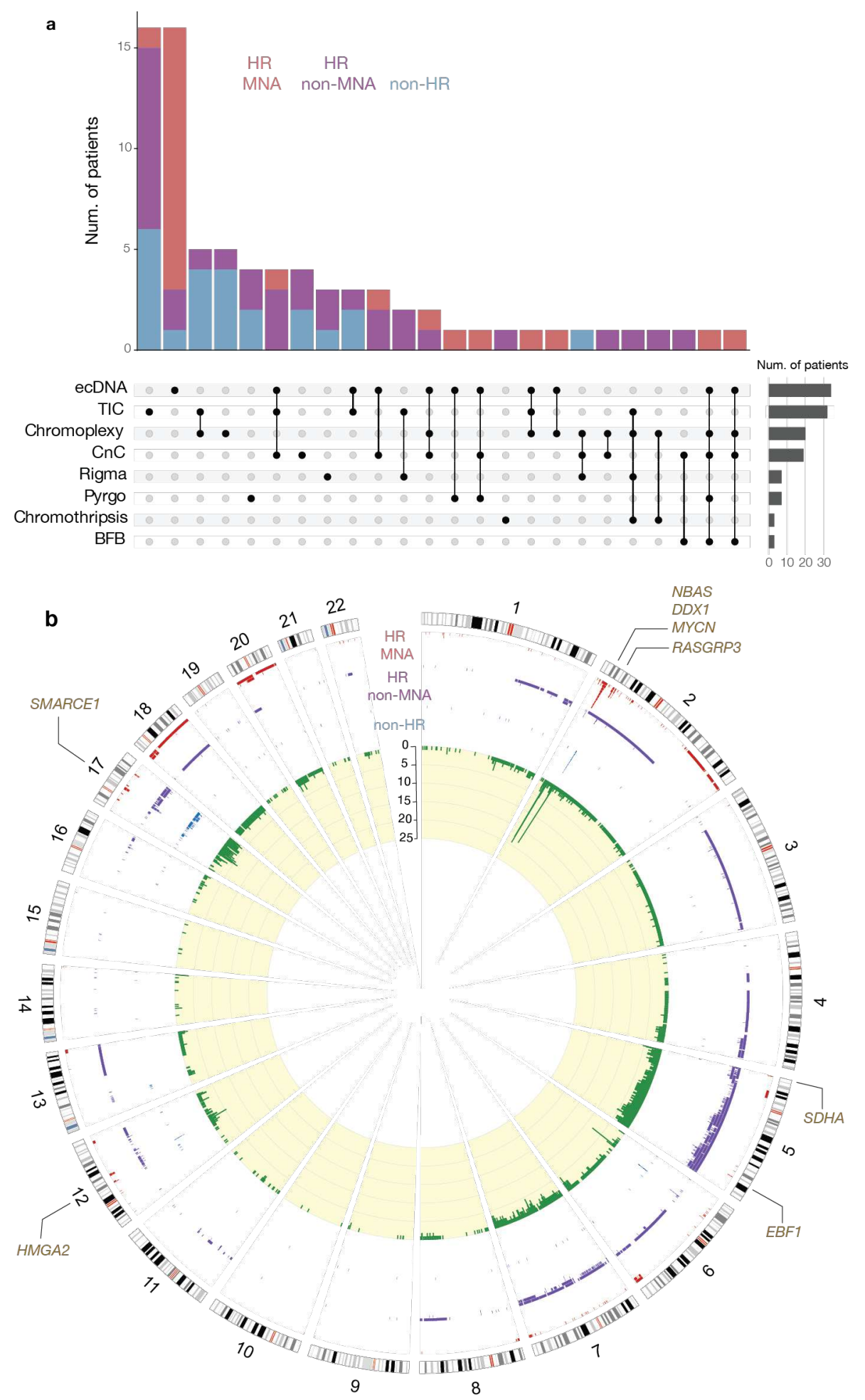

918 Figure 4. Topography of complex rearrangements in neuroblastoma. (a) Upset plot depicting 919 the co-occurrence of the different types of complex SV patterns within patients. The number of patients with each combination of rearrangements is shown in the top histogram (colors display the risk group for each patient). The histogram on the right panel displays the frequency for each of the complex SV types in our cohort. (b) Circos plot showing the regions of the genome involved in complex rearrangements in the three risk groups. The inner yellow circle shows the frequency of complex SVs in each region considering the whole neuroblastoma cohort $(\mathrm{n}=114)$. Scale is in percentage. MYCN, RASGRP3, SMARCE1, EBF1, SDHA, and $H M G A 2$ regions are highlighted as the most recurrently rearranged across patients. 

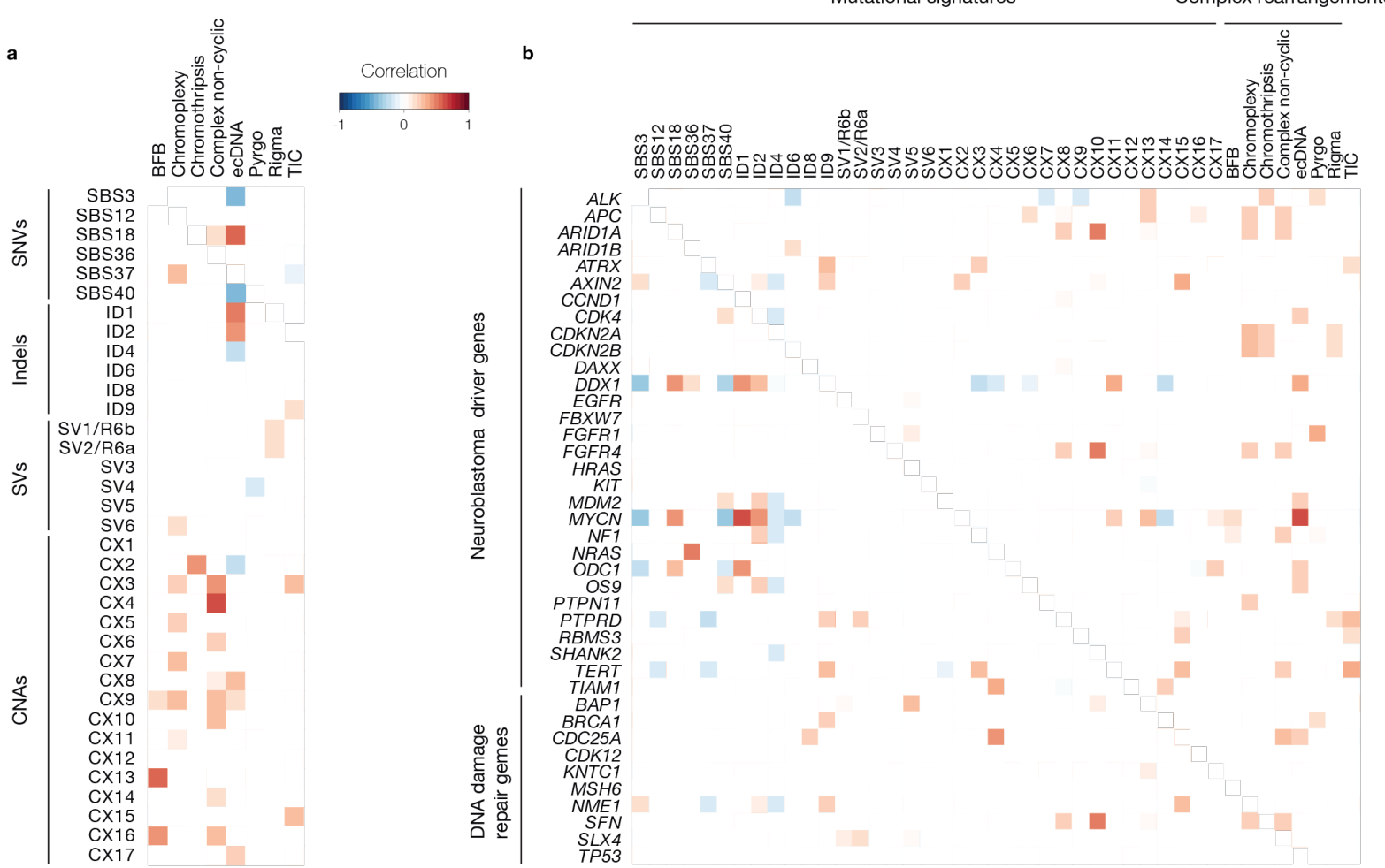

928 Figure 5. Co-occurrence of mutational signatures, complex rearrangements, and mutated

driver genes. (a) Heatmap depicting the correlations between the signatures associated with different variant types (SNV, indel, SV, and CNA) and the eight complex rearrangement classes identified in our cohort $(\mathrm{n}=114)$. Colors display the Spearman correlation coefficient. Only significant correlations are included ( $\mathrm{P}<0.05$, FDR correction). (b) Heatmap showing the correlations between mutated neuroblastoma driver genes and DNA damage repair genes and all the mutational signatures and complex rearrangement types identified in our cohort $(n=114)$. Colors display the Spearman correlation coefficient. Only significant correlations are included $(\mathrm{P}<0.05$, FDR correction). 
NEUROBLASTOMA MUTATIONAL SCENARIOS
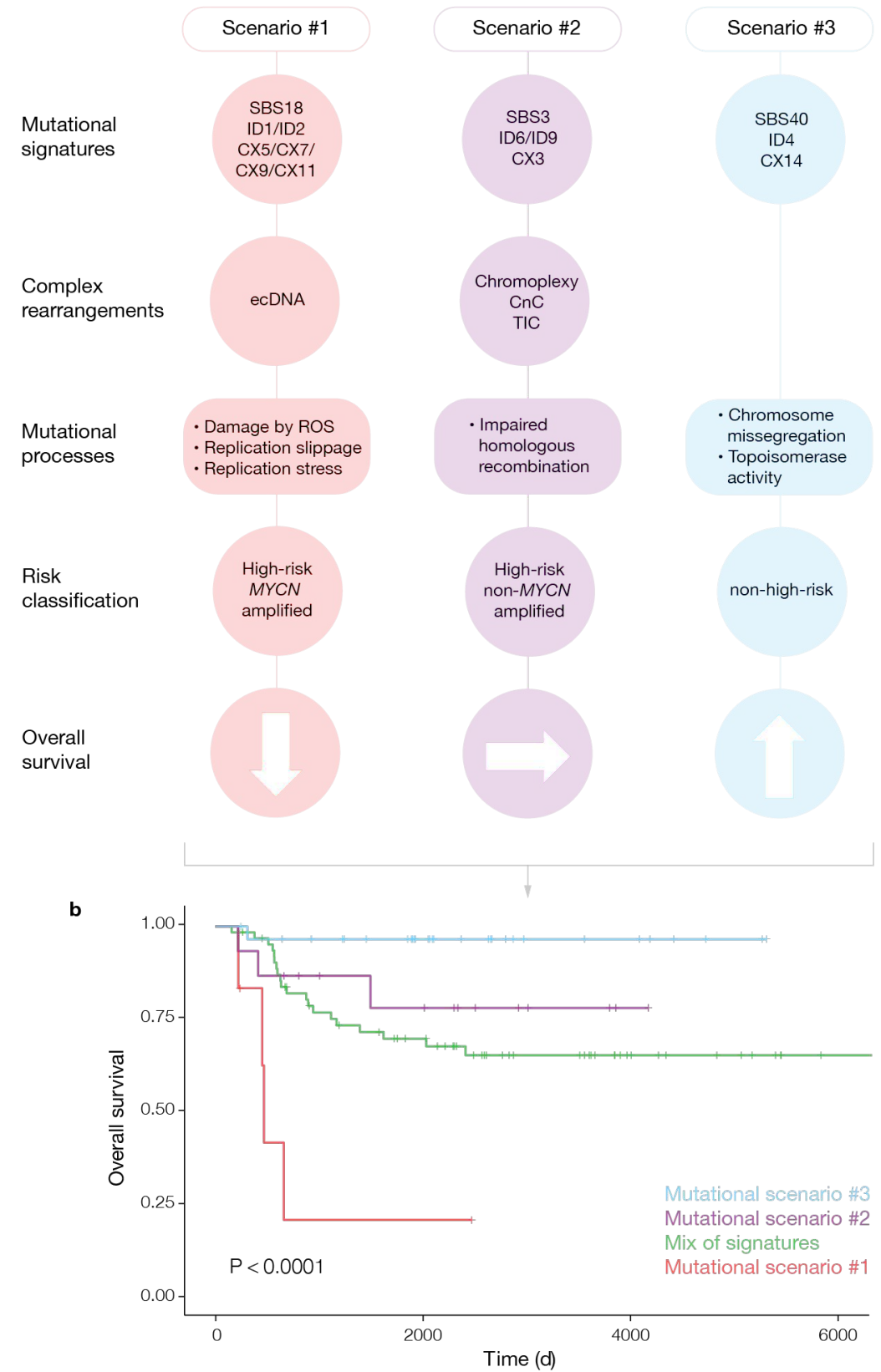

Figure 6. Clinical implications of different mutational processes and complex rearrangement patterns in neuroblastoma. (a) Summary of the three mutational scenarios defined in our neuroblastoma cohort, including the mutational processes, complex rearrangements, and clinical implications associated with each of them. (b) Kaplan-Meier survival curve showing the clinical impact of the three described mutational scenarios. Patients presenting all the signatures of scenario \#1 (SBS18-driven; red) have lower survival than patients with any mix of signatures not assigned to a single scenario (green), patients with all signatures of scenario \#2 (SBS3-driven; purple), and patients with all signatures of scenario \#3 (SBS40-driven; blue) $(\mathrm{P}=2.5 \times 10-2$ by Log-rank test). Colors on Kaplan-Meier plots display each condition. 


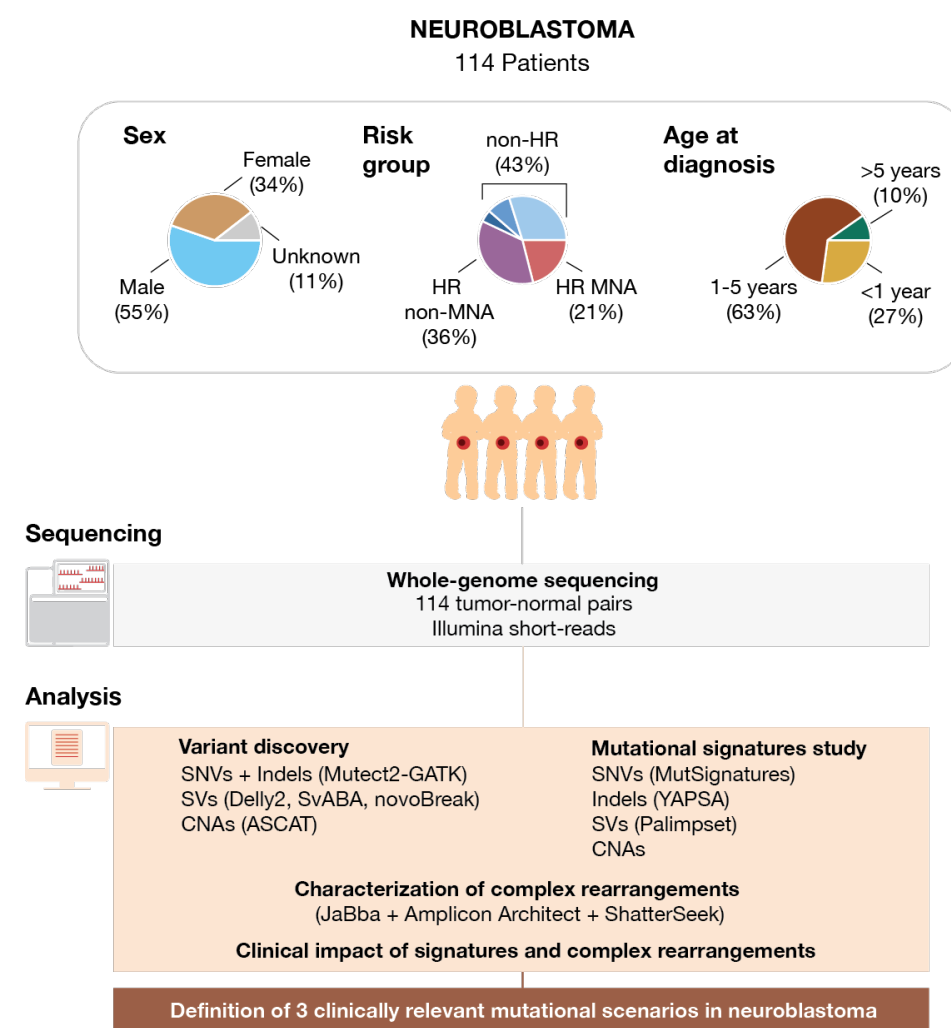

Supplementary Figure 1. Flow chart of the analysis strategy. Top, description of the distribution (in percentage) of the 114 neuroblastoma samples within sex groups (female, male, and unknown), risk groups (HR MNA, HR non-MNA, and non-HR: 29.8\% low-risk; 8.8\% low-risk stage $4 \mathrm{~S} ; 4.4 \%$ intermediate-risk), and age at diagnosis groups ( $<1$ year old, $1-5$ years old, and $>5$ years old). Middle, summary of the sequencing datasets available for our analysis. WGS matched tumor-normal pairs for 114 patients. Bottom, description of the main steps carried out in our study, starting by the variant discovery and the mutational signatures analysis, followed by the characterization of the complex rearrangements present in our samples, and the evaluation of the clinical impact of the identified mutational entities in neuroblastoma to finalize with the definition of the three clinically relevant neuroblastoma mutational scenarios presented in this work. 

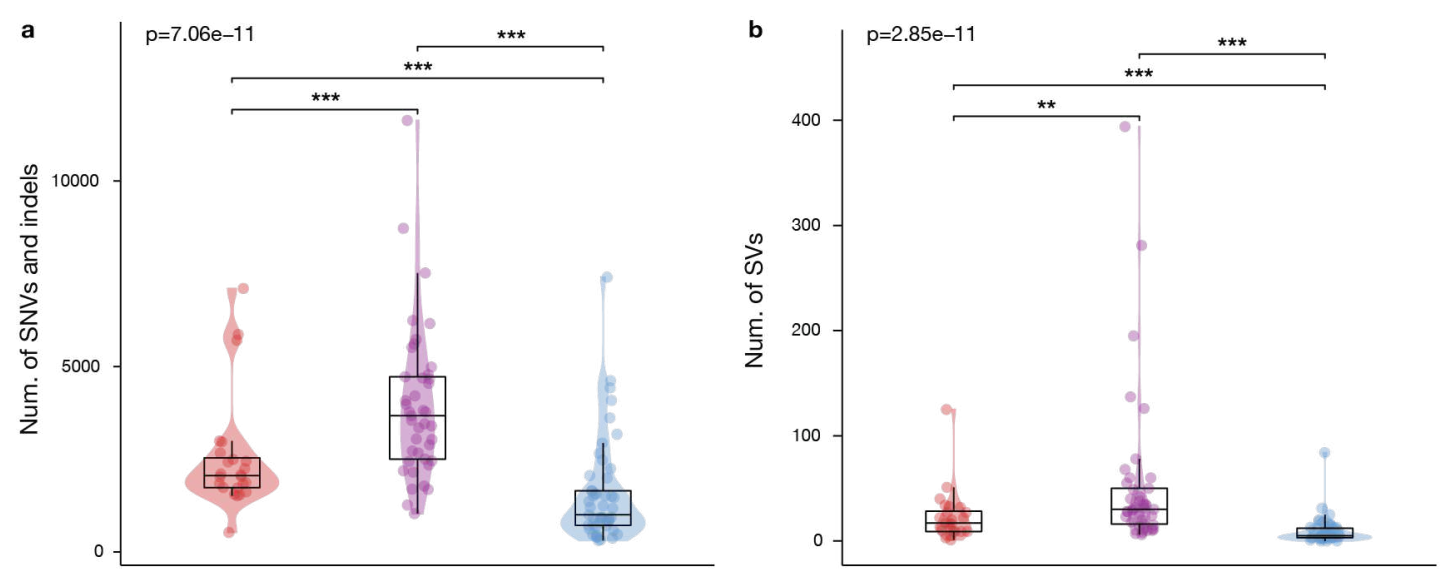

Risk groups: HR MNA

HR non-MNA non-HR

c

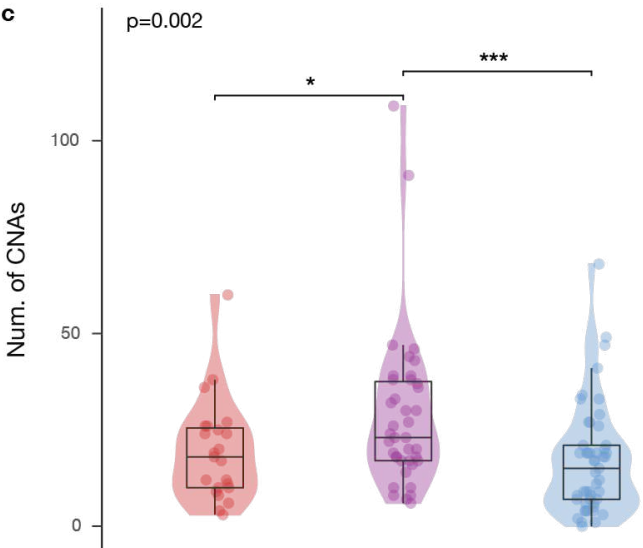

d

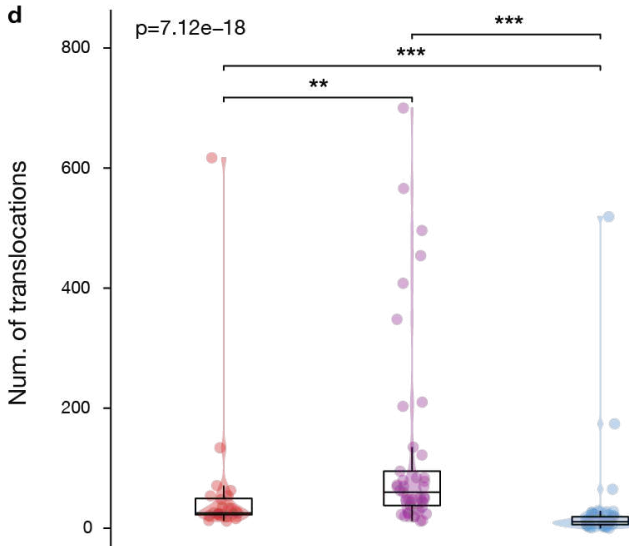

Supplementary Figure 2. Distribution of the different somatic variant types across the neuroblastoma risk groups. Comparison between distributions of different variant types in the three neuroblastoma risk groups (HR MNA, HR non-MNA, non-HR). To assess if there are differences between risk groups, we used the non-parametric Kruskal-Wallis test (p-value in the upper left corner). The pairwise comparisons were done using the non-parametric Wilcoxon rank-sum test. (a) SNVs and indels, (b) SVs, (c) CNAs, and (d) translocations. Significance legend: *: p-value $<0.1, * *$ : p-value $<0.05, * * *$ : p-value $<0.01$. 

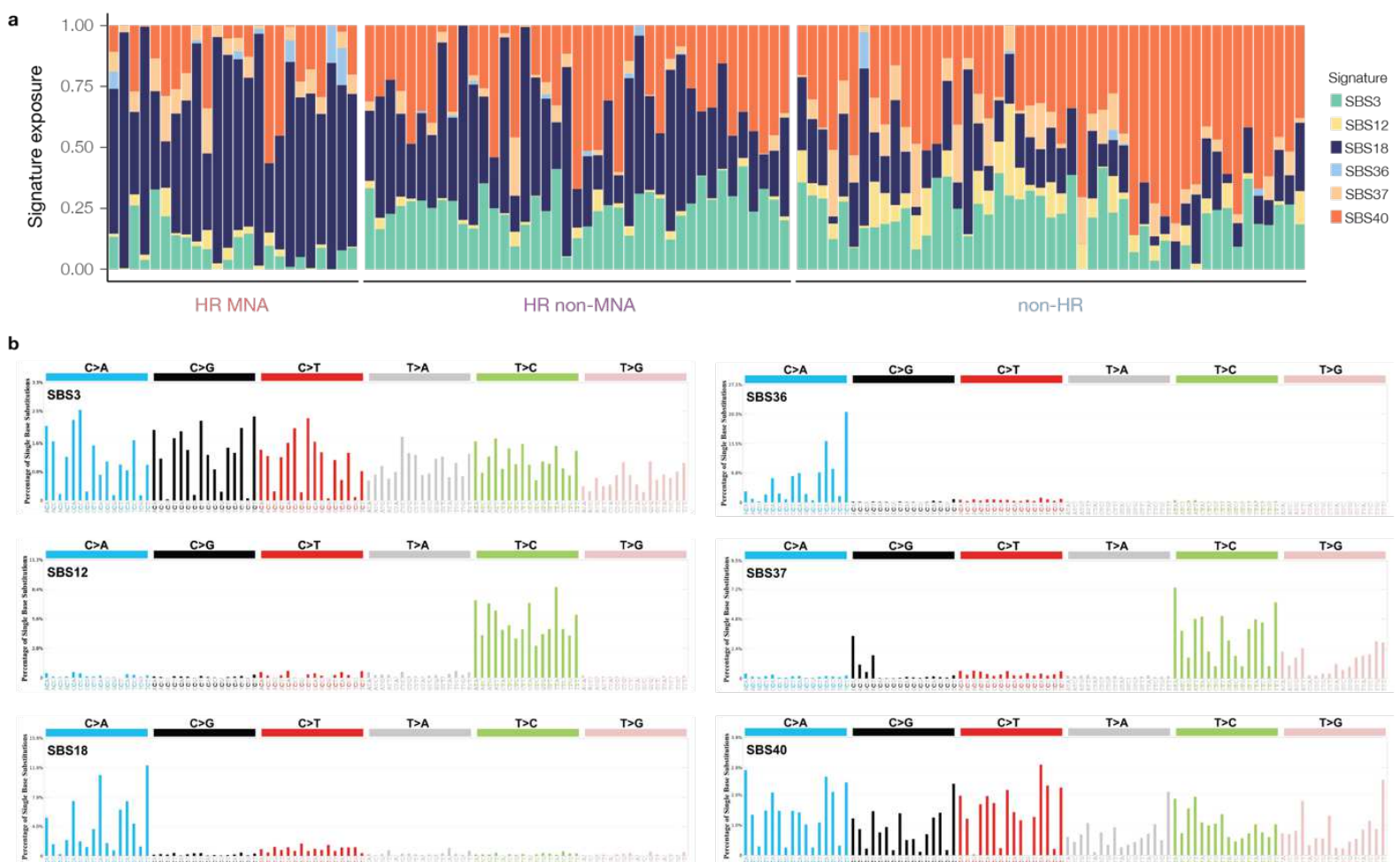

Supplementary Figure 3. SNV-based mutational signatures analysis. (a) Ratio of exposure of the six reference SNV-based signatures (SBS) identified in our cohort for each of the 114 neuroblastoma patients. Patients have been grouped by risk (HR MNA, HR non-MNA, nonHR). Each color displays a different signature: SBS3, SBS12, SBS18, SBS36, SBS37, SBS40. (b) Mutational profiles for each of the reference SNV-based signatures detected in our cohort, reproduced from the COSMIC database (https://cancer.sanger.ac.uk/signatures/sbs/). 

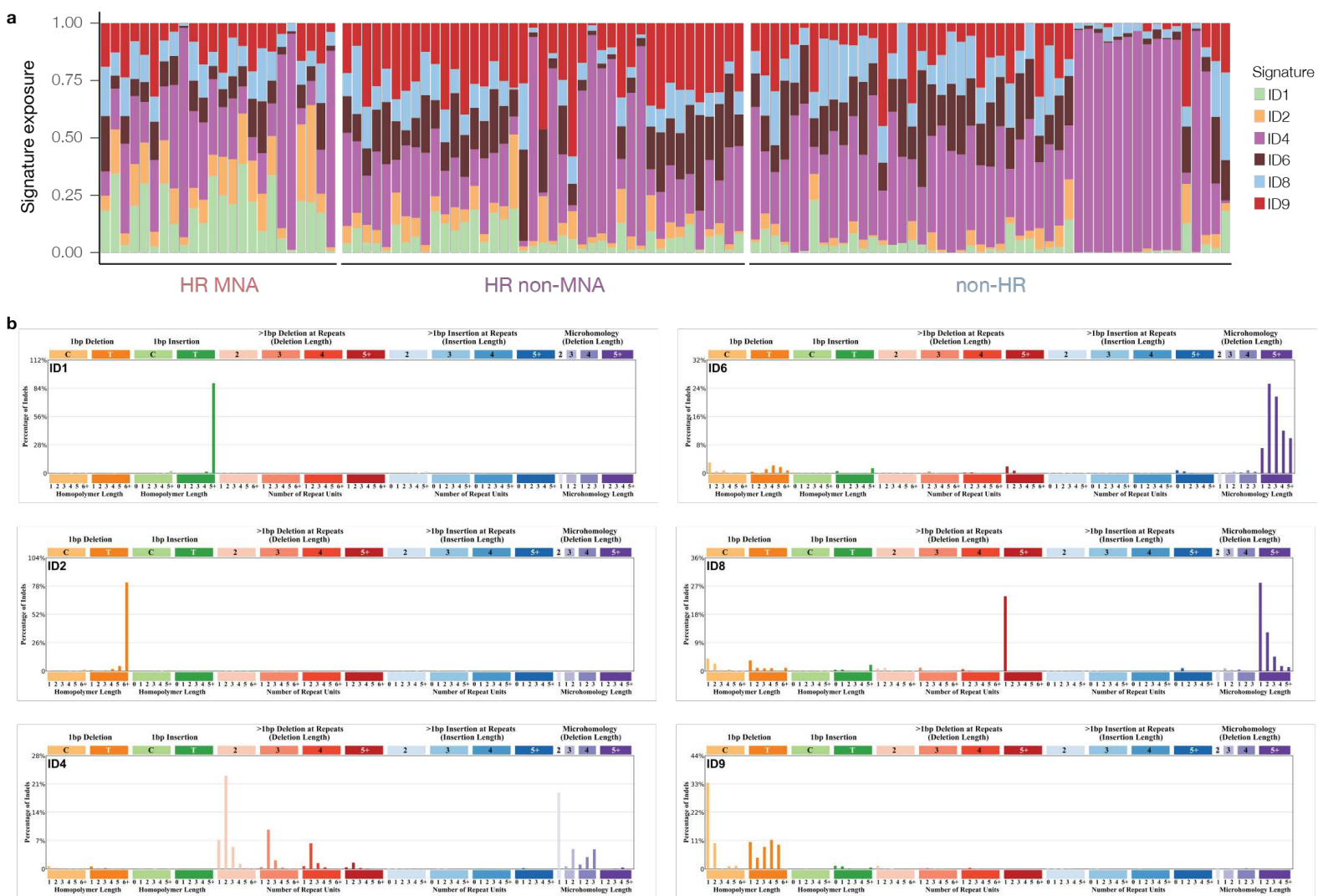

Supplementary Figure 4. Indel-based mutational signatures analysis. (a) Ratio of exposure of the six reference indel-based signatures (ID) identified in our cohort for each of the 114 neuroblastoma patients. Patients have been grouped by risk (HR MNA, HR non-MNA, nonHR). Each color displays a different signature: ID1, ID2, ID4, ID6, ID8, ID9. (b) Mutational profiles for each of the reference indel-based signatures detected in our cohort, reproduced from the COSMIC database (https://cancer.sanger.ac.uk/signatures/id/). 

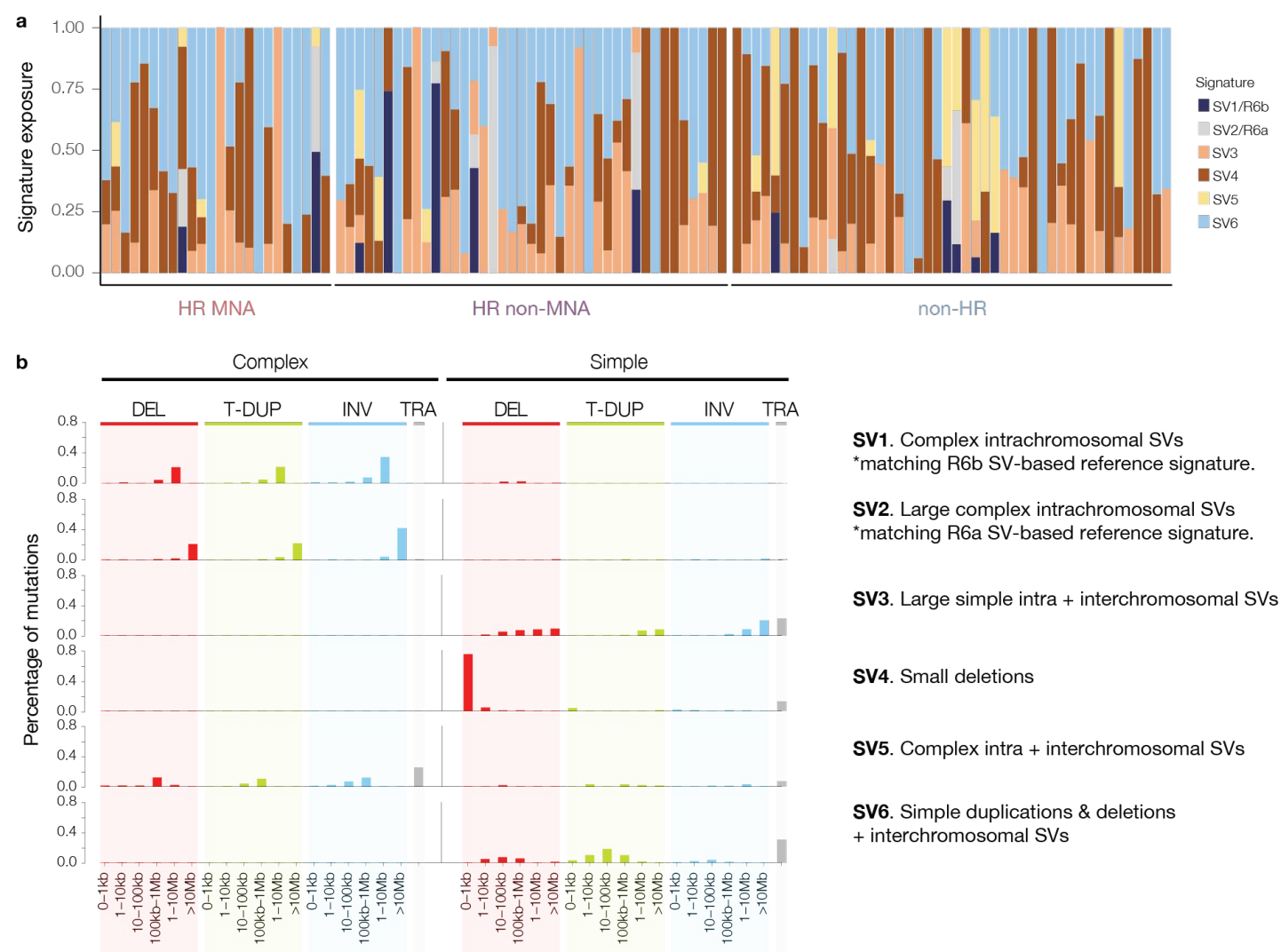

SV1. Complex intrachromosomal SVs *matching R6b SV-based reference signature.

SV2. Large complex intrachromosomal SVs *matching R6a SV-based reference signature.

SV3. Large simple intra + interchromosomal SVs

SV4. Small deletions

SV5. Complex intra + interchromosomal SVs

SV6. Simple duplications \& deletions + interchromosomal SVs
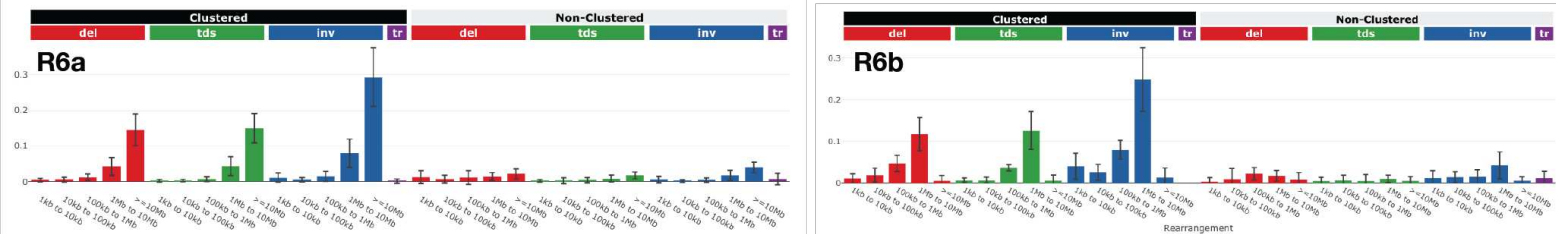

Supplementary Figure 5. SV-based mutational signatures analysis. (a) Ratio of exposure of the $6 \mathrm{SV}$-based signatures identified in our cohort for each of the 114 neuroblastoma patients. Patients have been grouped by risk (HR MNA, HR non-MNA, non-HR). Each color displays a different signature: SV1-SV6. (b) Mutational profiles for each of the SV-based signatures detected in our cohort. (c) Mutational profiles for reference SV-based signatures R6a and R6b, corresponding to the de-novo identified signatures SV2 and SV1, respectively, reproduced from Signal database (https://signal.mutationalsignatures.com/). 
1102

1103

1104

1105

1106

1107

1108

1109

1110

1111

1112

1113

1114

1115

1116

1117

1118

1119

1120

1121

1122

1123

1124

1125

1126

1127

1128

1129

1130

1131

1132

1133

1134

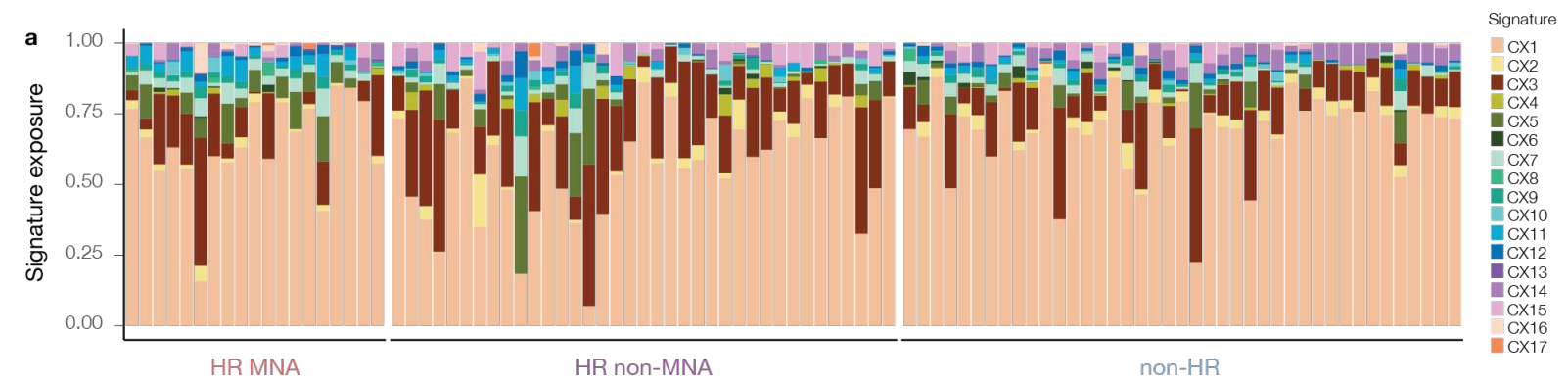

Supplementary Figure 6. CNA-based mutational signatures analysis. (a) Ratio of exposure of the 17 CNA-associated signatures identified in our cohort for each of the 114 neuroblastoma patients. Patients have been grouped by risk (HR MNA, HR non-MNA, non-HR). Each color displays a different signature: CX1-CX17. 

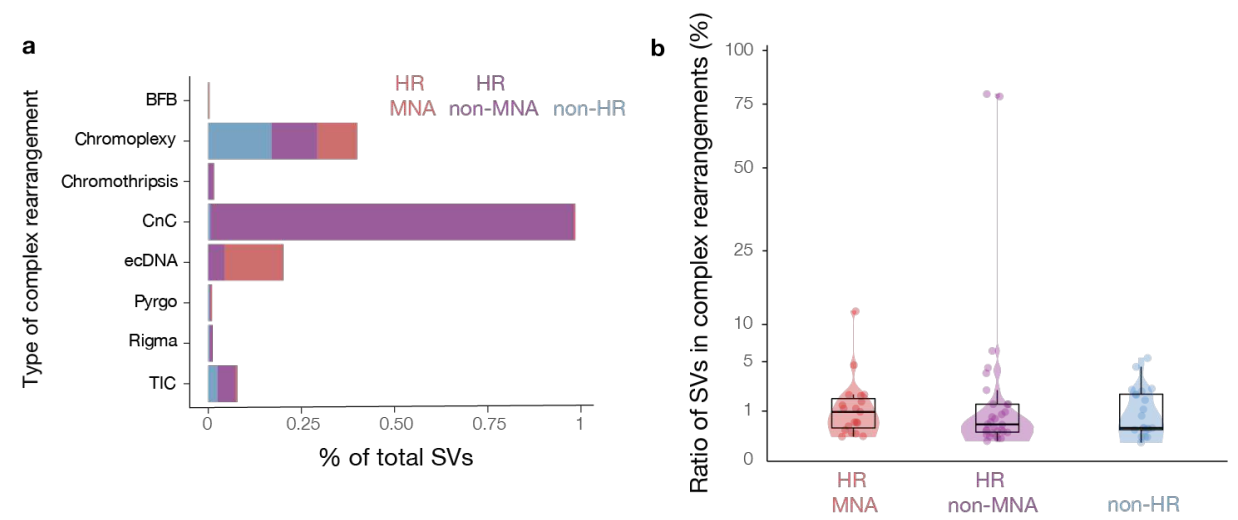

Supplementary Figure 7. General characteristics of complex rearrangements. (a) Percentage of SVs involved in each of the eight classes of complex rearrangement. Colors display the distribution of the rearrangements within neuroblastoma risk groups. (b) Comparison between distributions of the ratio of SVs involved in complex rearrangements across the three neuroblastoma risk groups (HR MNA, HR non-MNA, non-HR). To assess differences between risk groups, we used the non-parametric Kruskal-Wallis test. The pairwise comparisons were made using the non-parametric Wilcoxon rank-sum test. No significant differences were observed between risk groups. 

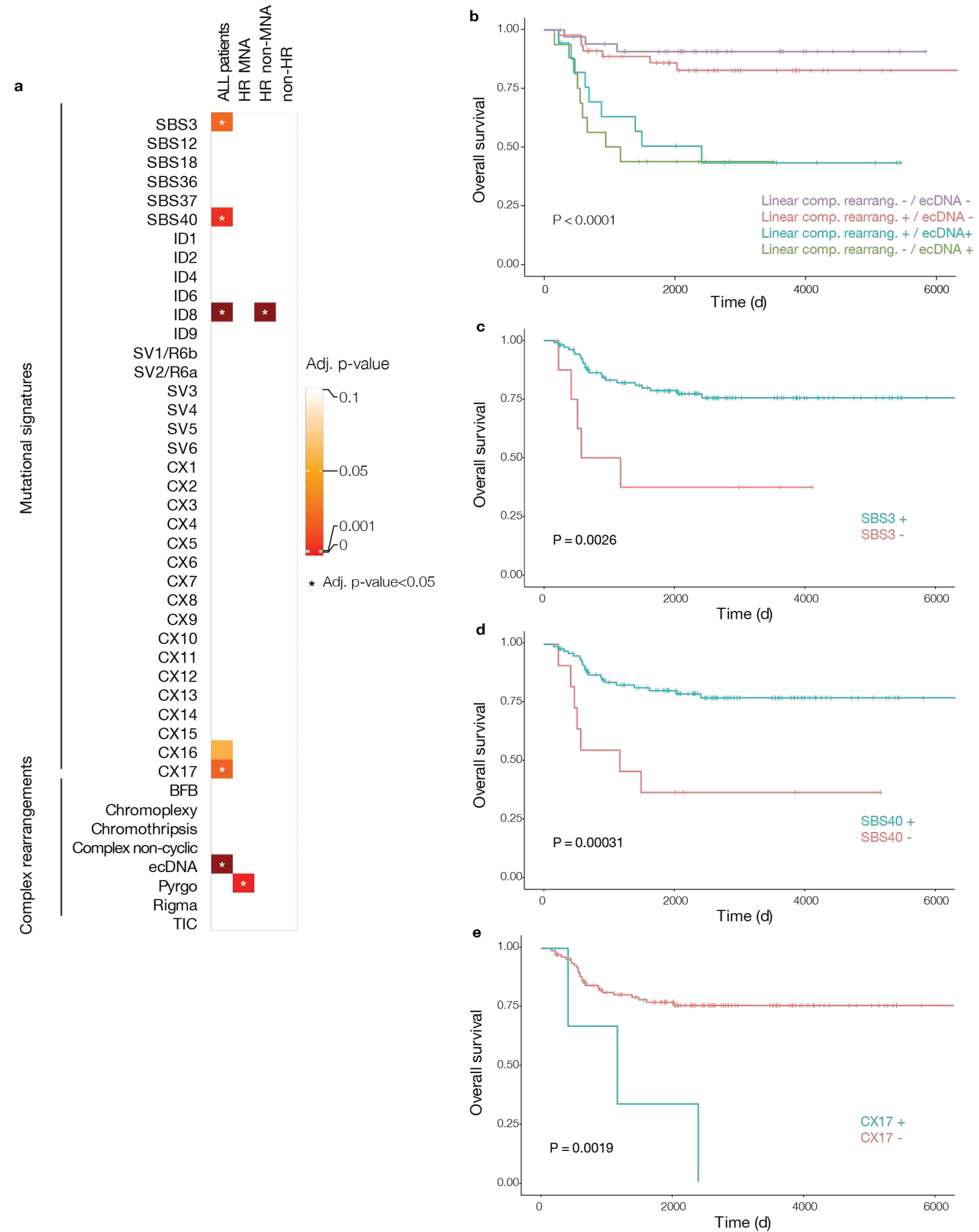

Supplementary Figure 8. Survival plots of mutational processes and complex rearrangements in neuroblastoma showing a significant clinical impact in neuroblastoma.

1178 (a) Heatmap showing the significant associations between the different mutational signatures and complex rearrangement types identified in our cohort $(n=114)$ with patients' overall survival. These multiple tests have been performed for all patients and each of the risk groups separately. P-values have been adjusted for multiple testing using false-discovery rate correction. Colors display the adjusted $p$-values $<0.1$. Significant associations are marked with an asterisk (adjusted p-value $<0.05$ ). Kaplan-Meier survival curve showing the overall survival of (b) patients presenting only ecDNA (green), ecDNA and linear complex rearrangements (blue), only linear complex rearrangements (turquoise) or no complex rearrangements (purple) ( $\mathrm{P}<1 \times 10-4$ by Log-rank test). (c) patients with $(\mathrm{n}=106)$ and without $(\mathrm{n}=8)$ exposure of 
1187 SNV-based signature SBS3. Patients exhibiting patterns of SBS3 (turquoise) have better 1188 survival than patients not showing this signature (red) $(\mathrm{P}<2.6 \times 10-3$ by Log-rank test). (d) 1189 patients with $(n=103)$ and without $(n=11)$ exposure of SNV-based signature SBS40. Patients 1190 exhibiting patterns of SBS40 (turquoise) have better survival than patients not showing this 1191 signature $(\mathrm{red})(\mathrm{P}<3.1 \times 10-4$ by Log-rank test). (e) patients with $(\mathrm{n}=111)$ and without $(\mathrm{n}=$ 1192 3) exposure of CNA-based signature CX17. Patients exhibiting patterns of CX17 (turquoise) 1193 have worst survival than patients not showing this signature (red) $(\mathrm{P}<1.9 \times 10-3$ by Log-rank 1194 1195 test). Colors on Kaplan-Meier plots display each condition. 
a

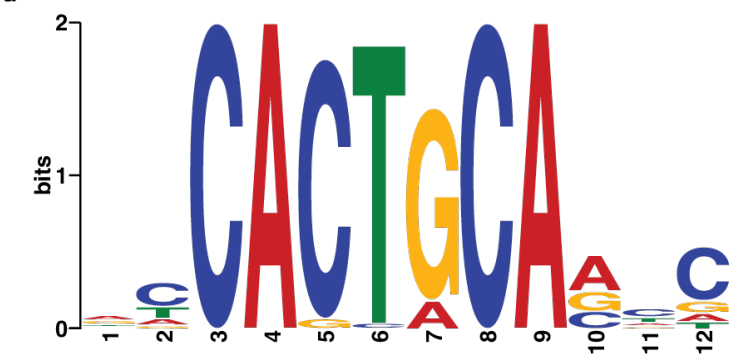

b

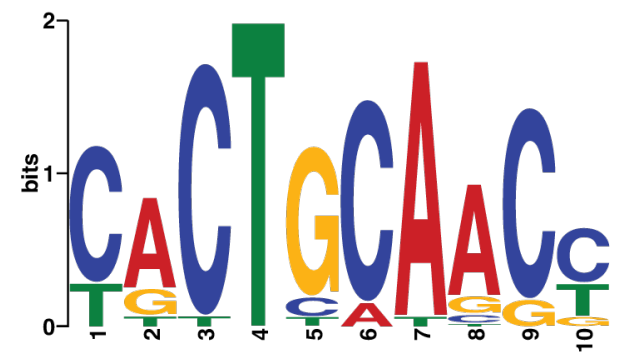

d

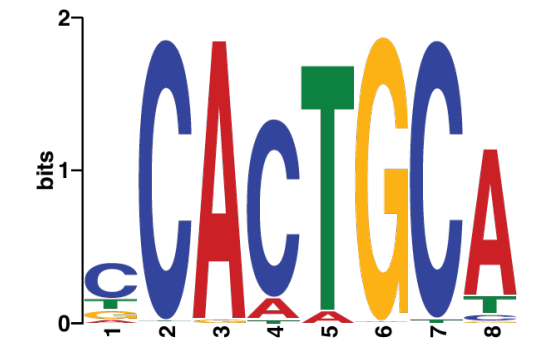

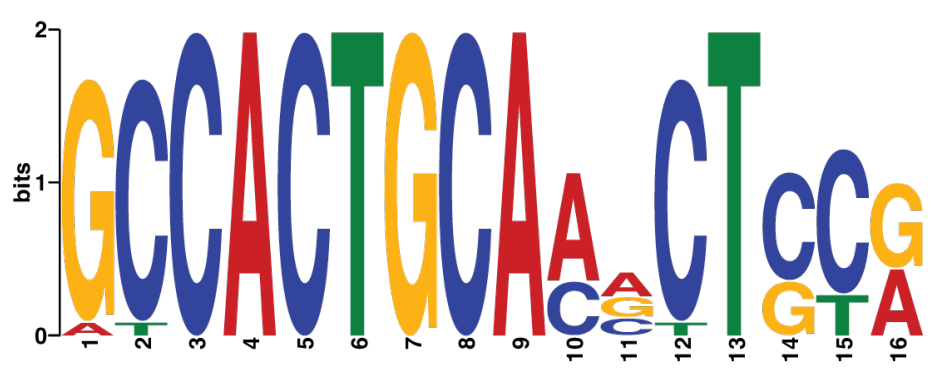

Supplementary Figure 9. Motifs closely resembling specific recombination signal sequences of PGBD5. Sequence logos detected near the SV breakpoints in (a) all 114 patients from our neuroblastoma cohort, (b) considering high-risk $M Y C N$-amplified patients exclusively, (c) considering high-risk non-MYCN-amplified patients exclusively, and (d) considering nonhigh-risk patients exclusively. 
Supplementary Table 1. Distribution of the exposure of each of the different mutational signatures across the three neuroblastoma risk groups. Table showing the exposure per risk group for each of the signatures detected in our neuroblastoma cohort. All numbers are percentages. The last column depicts the results of the Kruskal-Wallis rank sum test in order to evaluate the presence of significant differences in the exposure of each of the signatures across the three neuroblastoma risk groups. "yes" illustrates the presence of differences with a 1277 p-value $<0.05$.

\begin{tabular}{|c|c|c|c|c|c|}
\hline \multirow{2}{*}{ Signature ID } & \multirow{2}{*}{$\begin{array}{l}\text { Variant } \\
\text { type }\end{array}$} & \multicolumn{3}{|c|}{ Risk group exposure (\%) } & \multirow{2}{*}{$\begin{array}{l}\text { Differences } \\
\text { between risk groups }\end{array}$} \\
\hline & & HR MNA & HR non-MNA & non-HR & \\
\hline SBS3 & SNV & 9.2 & 25.4 & 21.7 & yes \\
\hline SBS12 & SNV & 2.4 & 1.4 & 6.9 & yes \\
\hline SBS18 & SNV & 63.4 & 40.1 & 19.3 & yes \\
\hline SBS36 & SNV & 2.2 & 0.3 & 0.5 & no \\
\hline SBSB37 & SNV & 7 & 2.4 & 8.1 & yes \\
\hline SBSB40 & SNV & 15.7 & 30.3 & 43.6 & yes \\
\hline ID1 & INDEL & 18.4 & 7.1 & 3.8 & yes \\
\hline ID2 & INDEL & 14.1 & 6.6 & 3.2 & yes \\
\hline ID4 & INDEL & 33.4 & 33.7 & 51.7 & yes \\
\hline ID6 & INDEL & 10.3 & 17.1 & 16 & yes \\
\hline ID8 & INDEL & 11.5 & 10.6 & 13.2 & no \\
\hline ID9 & INDEL & 12.3 & 24.9 & 12.1 & yes \\
\hline ID1+ID2 & INDEL & 32.5 & 13.7 & 7 & yes \\
\hline CX1 & CNA & 64.4 & 57.5 & 69.2 & yes \\
\hline $\mathrm{CX} 2$ & CNA & 1.75 & 2.39 & 2.76 & no \\
\hline $\mathrm{CX} 3$ & CNA & 12.2 & 21.5 & 12.8 & yes \\
\hline $\mathrm{CX} 4$ & CNA & 0.43 & 1.56 & 0.49 & no \\
\hline CX5 & CNA & 5.8 & 3.6 & 2.3 & yes \\
\hline CX6 & CNA & 0.3 & 0.17 & 0.5 & no \\
\hline $\mathrm{CX} 7$ & CNA & 3.7 & 2.5 & 2.4 & yes \\
\hline CX8 & CNA & 0.48 & 0.11 & 0.12 & no \\
\hline CX9 & CNA & 1.8 & 1.25 & 1.13 & yes \\
\hline CX10 & CNA & 0.68 & 0.58 & 0.31 & no \\
\hline CX11 & CNA & 3.25 & 1.33 & 0.83 & yes \\
\hline CX12 & CNA & 1.11 & 1.3 & 1.41 & no \\
\hline CX13 & CNA & 0.21 & 0.02 & 0.0002 & no \\
\hline CX14 & $\mathrm{CNA}$ & 1.38 & 2.17 & 3.77 & yes \\
\hline CX15 & CNA & 1.4 & 3.4 & 1.6 & no \\
\hline CX16 & CNA & 0.92 & 0.37 & 0.32 & no \\
\hline CX17 & CNA & 0.14 & 0.13 & 0 & no \\
\hline $\mathrm{CX} 7+\mathrm{CX} 9+\mathrm{CX} 11$ & CNA & 8.75 & 5.08 & 4.36 & yes \\
\hline SV1/R6b & SV & 2.8 & 5.9 & 1.8 & no \\
\hline SV2/R6a & SV & 2.8 & 4.2 & 1.8 & no \\
\hline SV3 & SV & 15.5 & 20.7 & 14.8 & no \\
\hline
\end{tabular}




\begin{tabular}{llllll}
\hline SV4 & SV & 29.9 & 25.7 & 39.8 & no \\
\hline SV5 & SV & 1.7 & 2 & 9.6 & no \\
\hline SV6 & SV & 47.2 & 41.6 & 32.1 & no \\
\hline SV1+SV2+SV5 & SV & 7.4 & 12 & 13.3 & no \\
\hline SV3+SV4+SV6 & SV & 92.6 & 88 & 86.7 & no \\
\hline
\end{tabular}

1278

1279

1280

1281

1282

1283

1284

1285

1286

1287

1288

1289

1290

1291

1292

1293

1294

1295

1296

1297

1298

1299

1300

1301

1302

1303

1304

1305

1306

1307

1308

1309

1310

1311

1312

1313

1314

1315

1316

1317

1318

1319

1320 
Supplementary Table 2. Distribution of the different complex rearrangement classes across the three neuroblastoma risk groups. (a) Table showing the percentage of complex rearrangements detected in our neuroblastoma cohort across the three different neuroblastoma risk groups. Numbers in brackets are absolute number of rearrangements. (b) Table showing the percentage of patients presenting complex rearrangements for each of the neuroblastoma risk groups. The last column depicts the results of the Kruskal-Wallis rank sum test in order to evaluate the presence of significant differences between neuroblastoma risk groups. "yes" illustrates the presence of differences with a p-value $<0.05$.

a)

\begin{tabular}{lllll}
\hline \multirow{2}{*}{ Complex SV class } & \multicolumn{3}{c}{ \# of complex SVs (\%) } & Differences \\
\cline { 2 - 4 } bFB & HR_MNA & \multicolumn{1}{c}{ HR_non-MNA } & non-HR & between risk groups \\
\hline Chromoplexy & $66.7(2)$ & $33.3(1)$ & 0 & no \\
\hline Chromothripsis & $13.9(5)$ & $55.6(20)$ & $30.5(11)$ & no \\
\hline CnC & 0 & $100(4)$ & 0 & no \\
\hline ecDNA & $34.3(12)$ & $57.1(20)$ & $8.6(3)$ & no \\
\hline Pyrgo & $68(53)$ & $25.6(20)$ & $6.4(5)$ & yes \\
\hline Rigma & $42.8(3)$ & $28.6(2)$ & $28.6(2)$ & no \\
\hline TIC & 0 & $71.4(5)$ & $28.6(2)$ & no \\
\hline
\end{tabular}

b)

\begin{tabular}{lllll}
\hline \multirow{2}{*}{ Complex SV class } & \multicolumn{3}{c}{ \# of patients with complex SVs (\%) } & Differences \\
\cline { 2 - 4 } & HR_MNA & HR_non-MNA & non-HR & between risk groups \\
\hline BFB & 8.3 & 2.4 & 0 & no \\
\hline Chromoplexy & 20.8 & 14.6 & 18.4 & no \\
\hline Chromothripsis & 0 & 7.3 & 0 & no \\
\hline CnC & 25 & 24.4 & 6.1 & no \\
\hline ecDNA & 100 & 22 & 6.1 & yes \\
\hline Pyrgo & 12.5 & 4.9 & 4.1 & no \\
\hline Rigma & 0 & 12.2 & 4.1 & no \\
\hline TIC & 12.5 & 41.5 & 24.5 & yes \\
\hline
\end{tabular}


Supplementary Table 3. FISH validation of the MYCN amplicon state in our cohort.

1346 Analysis of the MYCN amplicon state (homogenously stating region, HSR; extrachromosomal 1347 circular DNA, ecDNA) as measured by FISH and interpreted by the German clinical diagnostic 1348 reference center and tumor bank for neuroblastoma.

1349

\begin{tabular}{|c|c|c|}
\hline Sample ID & MYCN amplification & FISH confirmation \\
\hline CB2001 & Yes & ecDNA \\
\hline CB2013 & Yes & HSR/ecDNA \\
\hline CB2022 & Yes & ecDNA \\
\hline CB2024 & Yes & ecDNA \\
\hline CB2025 & Yes & HSR/ecDNA \\
\hline CB2027 & Yes & ecDNA \\
\hline CB2030 & Yes & ecDNA \\
\hline CB2031 & Yes & ecDNA \\
\hline CB2034 & Yes & HSR/ecDNA \\
\hline CB2040 & Yes & ecDNA \\
\hline CB2042 & Yes & ecDNA \\
\hline CB2043 & Yes & HSR \\
\hline CB2045 & Yes & ecDNA \\
\hline CB2050 & Yes & ecDNA \\
\hline NBL26 & Yes & ecDNA \\
\hline NBL27 & Yes & ecDNA \\
\hline NBL28 & Yes & ecDNA \\
\hline NBL29 & Yes & ecDNA \\
\hline NBL30 & Yes & ecDNA \\
\hline NBL31 & Yes & ecDNA \\
\hline NBL32 & Yes & ecDNA \\
\hline NBL33 & Yes & ecDNA \\
\hline NBL34 & Yes & ecDNA \\
\hline NBL42 & Yes & ecDNA \\
\hline
\end{tabular}

